\title{
EL PROCESO DE REORGANIZACIÓN CONCURSAL DE ESTADOS UNIDOS (CHAPTER ELEVEN DEL UNITED STATES CODE). COMPARACION CON LOS PROCEDIMIENTOS EXTRAJUDICIALES DE REORGANIZACIÓN
}

Héctor José Miguens ${ }^{1}$

\section{Resumen}

El presente trabajo estudia una parte del sistema concursal de los Estados Unidos que abarca las soluciones preventivas de la quiebra en ese país, que pretende preservar y virtualmente salvar a la empresa de su desaparición económica y jurídica y es conocido por el nombre de su respectivo capítulo, el Chapter Eleven o Capítulo Once del Title Eleven del United States Bankruptcy Code, una norma concursal federal que engloba numerosas materias en sus distintos capítulos.

El Chapter Eleven tiene por objeto establecer un proceso concursal de "reorganización" de la empresa en crisis o en estado de cesación de pagos ("Reorganization Procedure") a fin de sanearla económica o financieramente, solucionar el problema económico o financiero, facilitar su continuación empresarial y así librarla de su liquidación judicial forzosa, solución última reglada en el Chapter Seven de ese Código Concursal federal.

Este trabajo constituye una comparación entre el Chapter Eleven y los procesos extrajudiciales de reorganización empresarial y reestructuración de deuda en los Estados Unidos.

Al respecto este trabajo aborda los temas relativos a la elección alternativa de ambos procedimientos, sus ventajas e inconvenientes, los distintos procedimientos extrajudiciales estatales no federales del país de reestructuración de deuda, el management de la empresa en crisis, la negociación con los principales prestamistas con preferencias, la negociación con los pequeños acreedores y la utilización de especiales técnicas de financiación postconcursal.

En la parte conclusiva se realiza una valoración crítica de estas instituciones.

Palabras clave: derecho concursal - reorganización empresarial - capítulo once del Código de Bancarrotas de Estados Unidos - procedimientos extrajudiciales - procedimientos preventivos de la quiebra

\section{TERMINOLOGÍA UTILIZADA. ADVERTENCIAS PRELIMINARES}

He procurado mantener los vocablos y expresiones típicas del derecho concursal y societario estadounidense en inglés. Por ello en muchos casos no han sido traducidas al castellano para brindar un exacto

\footnotetext{
${ }^{1}$ Abogado (Universidad Nacional de Buenos Aires), Especialista en Sindicaturas Concursales (Universidad Nacional de Tucumán), Doctor en Derecho, (Universidad de Navarra, España). Investigador Independiente del CONICET (Consejo Nacional de Investigaciones Científicas y Técnicas), Argentina. Profesor Asociado de Derecho Concursal, Facultad de Derecho, Universidad Austral, Buenos Aires. Este trabajo ha sido realizado con el apoyo económico del CONICET. E-mail: hmiguens@gmail.com
} 
panorama terminológico propio del derecho concursal (federal) y del derecho societario (mayoritariamente local o estatal) en los Estados Unidos. En su caso he provisto su correspondiente explicación.

Cuando hablo de derecho "norteamericano", "americano" o "estadounidense" me refiero al derecho concursal federal de Estados Unidos. Mientras que las normas concursales de ese país son federales, las normas de derecho societario aplicables al caso concursal son predominantemente locales, pertenecientes a cada uno de los cincuenta Estados. Asimismo quedan englobadas las distintas instituciones de cada Estado sobre negociación de deuda y sobre reorganización extrajudicial empresarial.

Cuando hablo de "Reorganización", Chapter Eleven", Capitulo once", "Chapter 11", etc. me estoy refiriendo al proceso de "Reorganization" legislado en el "Chapter Eleven" o "Capítulo Once" del United States Bankruptcy Code (U.S.C., por sus siglas en inglés). Las normas referidas al Chapter 11 del U.S. Bankruptcy Code, serán enunciadas como U.S.C. precedido del número 11 ( o el número del capitulo que corresponda citar perteneciente a los restantes capítulos de ese cuerpo normativo, cuyo acronismo es U.S.C.), seguido del párrafo, parágrafo, "Section" o artículo correspondiente.

Por la “1994 Amendments" me refiero al Bankruptcy Reform Act de 1994, Pub. L. número 103-394, 108 Stat. 4106, codificado en algunas Sections dispersas en 11 U.S.C., del 18 U.S.C. y del 28 U.S.C.

Por las "2005 Amendments" me refiero al denominado Bankruptcy Abuse Prevention and Comsumer Protection Act de 2005, Pub. L. número 109-8, 119 Stat. 23, codificado en algunas Sections dispersas en 11 U.S.C., 18 U.S.C. 28 U.S.C.

"Bankruptcy Code" es el Bankruptcy Reform Act de 1978, Pub. L. número 95-598, 92 Stat. 2549, codificado en 11 U.S.C. \$\$101-1532, reformado el 20 de abril de 2005.

"Exchange Act" significa la Securities Exchange Act de 1934, ch. 404, 48 Stat. 881, codificado en 15 U.S.C. \$\$ 78a-78mm, modificado el 15 de mayo de 2006.

“IRC" significa el Internal Revenue Code de 1986, Pub. L. número 99-514, 100 Stat. 2085, codificado en 26 U.S.C. \$\$1-9833, modificado el 31 de diciembre de 2005.

"SEC" significa la Securities and Exchange Commission, órgano de aplicación de los títulos denominados "securities".

"Securities Act" significa la Securities Act de 1933, ch. 38, 48 Stat. 74, codificado en 15 U.S.C. \$\$77a-77aa, modificado el 15 de mayo de 2006.

El término "Statutes" ha sido traducido como "estatutos" o "normas estatutarias". Se refiere a cuerpos normativos específicos de derecho sancionados por los distintos Estados de la Unión o bien por el gobierno federal, que forman parte del plexo normativo aplicable conjuntamente con las sentencias judiciales, sean estatales o federales. 
He seguido la forma norteamericana de citar la legislación, doctrina y la jurisprudencia. Esta forma de citar (abreviaturas de los títulos de las revistas, etc.) está tomada del Bluebook ${ }^{2}$, normativa de citación aceptada en todo el país, en el que se explican sus elementos.

El término "Section" se refiere a las normas contenidas en estatutos normativos específicos, lo que equivale a "artículo" de nuestros ordenamientos. En su caso he indicado con un número seguido por un punto, como es usual en el sistema norteamericano, o bien mediante el signo de parágrafo (\$).

\section{INTRODUCCIÓN. OBJETO DE ESTE TRABAJO}

Las empresas pueden encontrarse en una situación de bonanza económico-financiera (es decir "in bonis") o bien tener dificultades financieras transitorias o perder la sustentabilidad económica. Para solucionar estos problemas los operadores jurídicos han ido perfilando los ordenamientos concursales a lo largo del tiempo. Uno de los sistemas concursales más proficuos, eficaces y pragmáticos lo constituye el sistema concursal norteamericano.

En el presente trabajo haré referencia tangencialmente a una parte de ese ordenamiento concursal de Estados Unidos, que abarca las soluciones preventivas de la quiebra que pretende salvar a la empresa de su desaparición económica y jurídica y es conocido por el nombre de su respectivo capítulo, el Chapter Eleven o Capítulo Once del Title Eleven del United States Code, una parte de la norma concursal federal que engloba numerosas materias en sus distintos capítulos ${ }^{3}$.

\footnotetext{
${ }^{2}$ Harvard Law Review Association (Ed.). The Bluebook. A uniform system of Citation. 19 Ed. Cambrigde. Massachusetts, 2003. Existen ediciones posteriores.

${ }^{3}$ Para una bibliografía general sobre el proceso concursal del Chapter Eleven en los Estados Unidos, pueden consultarse la siguiente, entre otros autores: AAVV. WEIL, GOTSHAL \& MANGES, LLP, Reorganizing Failing Businesses, A Comprehensive Review and Analysis of Financial Restructuring and Business Reorganization, Rev. Edition, American Bar Association, Chicago, 2006 (Dos volúmenes); Charles J. TABB, “The Future of Chapter 11”, 44 S. C. L. Rev. 791, 804 (1993); William D. WARREN / Daniel J. BUSSEL, Bankruptcy, University Casebook Series, 4th ed., Foundation Press, New York, 2002; Elizabeth WARREN, Chapter 11: Reorganizing American Businesses, Aspen Publishers, Wolters Kluwer Law \& Business, Austin / Boston / Chicago / New York / The Netherlands, 2008; Elisabeth WARREN / Jay Lawrence WESTBROOK, The Law of Debtors and Creditors, Text, Cases and Problems, Wolters Kluwer Law and Business, Austin / Boston / Chicago / New York / The Netherlands, $6^{\text {th }}$ Ed., 2009; AAVV, Alan B. MORRISON (ed.), Fundamentals of American Law, Oxford University Press, Oxford, 1997; Jeff FERRIELL / Edward J. JANGER, Understanding Bankruptcy, Lexis Nexis, $2^{\text {nd }}$. Ed, New York, 2007; Charles J. TABB, Bankruptcy Anthology, Anderson Publishing Co., Cincinnati, Ohio, 2002; Margaret HOWARD, Bankruptcy, Cases and Materials, American Casebook Series, Thomson / West Publishing, St. Paul, Minnesota, 2004; Teresa SULLIVAN / Elisabeth WARREN / Jay L. WESTBROOK, As We Forgive our Debtors: Bankruptcy and Consumer Credit in America, Oxford University Press, Oxford, 1989 (hay edición 1991); Theodore EISENBERG, "Bankruptcy Law in Perspective”, 28 UCLA L. Rev. 953 (1981); Thomas H. JACKSON, The logic and limits of bankruptcy law, Harvard University Press, Boston, 1986; Philip BLUMBERG, Problems in the bankruptcy or reorganization of parent and subsidiary corporations, including the law of corporate guaranties, Little, Brown \& Co., Boston, 1985 y supplement 2000 ; AAVV., David S. CLARK / Turgrul ANSAY (eds.), Introduction to the Law of the United States, Kluwer Law International, The Hague / London / New York, 2002; Michael J. HERBERT, Understanding Bankruptcy, Lexis Nexis, Matthew Bender, New York, 1995; Charles J. TABB / Ralph BRUBAKER, Bankruptcy Law: Principles, Policies and Practice, Lexis Nexis, $2^{\text {nd }}$ Ed., 2006; Mark J. ROE, Bankruptcy and Corporate Reorganization, Legal and Financial Materials, Foundation Press, New York, 2007; Mark S. SCARBERRY / Kenneth N. KLEE / Grant W. NEWTON / Steve H. NICKES, Business Reorganization in Bankruptcy, Cases and Materials, Thomson / West Publishing, $3^{\text {rd }}$. Ed., 2006; Douglas G. BAIRD / vol.09, nº. 01, Rio de Janeiro, 2016.pp. 545-585 
Existen obras que tratan aspectos particulares del Chapter Eleven ${ }^{4}$ y obras que hacen críticas (especialmente desde la cosmovisión del "Law and Economics" approach) y han propuesto reformas a esta parte del Código Concursal 5 . En contraste, otros autores son claros defensores de este proceso concursal ${ }^{6}$. Luego de un

Thomas H. JACKSON / Barry E. ADLER, Bankruptcy, Cases, Problems and Materials, University Casebook Series, Rev. Foundation Press, New York, $3^{\text {rd }}$. Ed., 2001; Michael J. CRAMES / Herbert S. EDELMAN / Andrew A. KRESS, The Fundamentals of Bankruptcy and Corporate Reorganization, Juris Publishing, 1998; Charles Jordan TABB, The Law of Bankruptcy, The Foundation Press, Westbury, New York, 1997; Martin A. FREY, Introduction to Bankruptcy Law, Thomson / Delmar Learning, 2007; Douglas G. BAIRD, The Elements of Bankruptcy, Foundation Press, New York, $4^{\text {th }}$ Ed., 2006; Brian A. BLUM, Bankruptcy and Debtor / Creditor, Examples and Explanations, Aspen Publishers, New York, 2006; Bankruptcy Code, Rules \& Official Forms, 2005 Law School Edition, Thomson West; Alan N. RESNICK, Bankruptcy Law Manual, Thomson / West, $5^{\text {th }}$. Ed., 2002-; Lewis KRUGER, Understanding the Basics of Business Bankruptcy and Reorganization 2008, Practicing Law Institute, New York, 2000 et sig.; BHANDARI, Jagdeep S., Corporate Bankruptcy: Economic and Legal Perspectives, New York, 1996; este autor, "El proceso de Reorganización Concursal de Estados Unidos. (Chapter Eleven del U. S. Code). Introducción y aspectos generales”, en Anuario de Derecho Concursal (España), vol 34 (enero 2015) páginas 477-543.

${ }^{4}$ Sobre estos temas particulares pueden consultarse, entre otros autores: Theodore EISENBERG, "The Unsecured Creditor in Reorganizations and the Nature of Security", 38 Vand. L. Rev. 931 (1985); Theodore EISENBERG \& Lynn LOPUCKI, "Shopping For Judges: An Empirical Analysis of Venue Choice in Chapter 11 Reorganizations", 84 Cornell L. Rev. 967 (1999); Kathryn SMYSER, "Going Private and Going Under: Leveraged Buyouts and the Fraudulent Conveyance Problem”, 63 Ind. L. J. 781 (1988); Ralph BRUBAKER, "One Hundred Years of Federal Bankruptcy Law and Still Clinging To An In Rem Model of Bankruptcy Jurisdiction”, 15 Bankr. Dev. J. 261 (1999); Daniel KEATING, “Offensive Uses of the Bankruptcy Stay”, 45 Vand. L. Rev. 71 (1991); Douglas G. BAIRD, "Revisiting Auctions in Chapter 11”, 36 J. L. \& Econ. 633 (1993); Alan SCHWARTZ, "Contracting About Bankruptcy”, 13 J. L. \& Econ. 595 (1993); Alan SCHWARTZ, "Bankruptcy Workouts and Debt Contracts", 36 J. L. \& Econ. 595 (1993); Lynn M. LOPUCKI, “Contract Bankruptcy: A Reply to Alan Schwartz”, 109 Yale L. J. 317 (1999); Steven L. SCHWARCZ, "Rethinking Freedom of Contract: A Bankruptcy Paradigm”, 77 Tex. L. Rev. 515 (1999); Lynn M. LOPUCKI, “Bankruptcy Contracting Revised: A Reply to Alan Schwartz’s New Model”, 109 Yale L. J. 365 (1999);

${ }^{5}$ Para esto pueden consultarse los siguientes autores, entre otros: Michael BRADLEY \& Michael ROSENZWEIG, "The Untenable Case for Chapter 11”, 101 Yale L.J. 1043 (1992); Robert K. RASMUSSEN, “Debtor’s Choice: A Menu Approach to Corporate Bankruptcy”, 71 Tex. L. Rev. 51 (1992); Barry Ed. ADLER, “A World Without Debt”, 72 Wash. U. L. Q. 811 (1994); Barry Ed. ADLER, "Finance’s Theoretical Divide and the Proper Role of Insolvency Rules”, 68 S. Cal. L. Rev. 401 (1994); John D. AYER, “Chapter 11: Uses and Consequences”, 4 Am. Bankr. Inst. L. Rev. 493 (1996); James W. BOWERS, “Groping and Coping in the Shadow of Murphy's Law: Bankruptcy Theory and the Elementary Economics of Failure”, 88 Mich. L. Rev. 2097 (1990); Robert K. RASMUSSEN, “The Efficiency of Chapter 11”, 8 Bankr. Dev. J. 319 (1991); Mark J. ROE, "Bankruptcy and Debt: A New Model for Corporate Reorganizations”, 83 Colum. L. Rev. 527 (1983); Mark E. MACDONALD et al., "Chapter 11 as a Dynamic Evolutionary Learning Process in a Market with Fuzzy Values”, 1993-1994 Ann. Surv. Bankr. L. 1; Theodore EISENBERG \& Shoichi TAGASHIRA, "Should We Abolish Chapter 11? The Evidence From Japan”, 23 J. Legal Stud. 111 (1994); J. Bradley JOHNSTON, “The Bankruptcy Bargain”, 65 Am. Bankr. L. J. 213 (1991); Edith A. JONES, "Chapter 11: A Death Penalty for Debtor and Creditor Interests”, 77 Cornell L. Rev. 1088 (1992); Michelle J. WHITE, "Corporate Bankruptcy as a Filtering Device: Chapter 11 Reorganiations and Out-of-Court Deb Restructurings”, 10 J. L. Econ. \& Org. 268 (1994); Kenneth N. KLEE, "Adjusting Chapter 11: Fine Tuning the Plan Process", 69 Am. Bankr. L. J. 551 (1995); Philippe AGHION, Oliver HART \& John MOORE, “The Economics of Bankruptcy Reform”, 8 J. L. Econ. \& Org. 523 (1992); Lisa H. FENNING, “The Future of Chapter 11: One View from the Bench”, 1993-1994 Ann. Surv. Bankr. L. 113; Michelle J. WHITE, "Does Chapter 11 Save Economically Inefficient Firms?” 72 Wash. U.L.Q. 1319 (1994); Barry E. ADLER, "Financial and Political Theories of American Corporate Bankruptcy”, 45 Stan. L. Rev. 311 (1993); Barry E. ADLER, "Bankruptcy and Risk Allocation”, 77 Cornell L. Rev. 439 (1992); Philippe AGHION et al., "The Economics of Bankruptcy Reform”, J.L. Econ \& Organization 523 (1992); Robert K. RASMUSSEN, “The Ex Ante Effects of Bankruptcy Reform on Investment Incentives”, 72 Wash U.L.Q. 1159 (1994); Lynn M. LOPUCKI, "Chapter 11: An Agenda for Basic Reform”, 69 Am. Bankr. L. J. 573 (1995); Lucian Ayre BEBCHUK, “A New Approach to Corporate Reorganizations”, 101 Harv. L. Rev. 775 (1986); James W. BOWERS, "Rehabilitation, Redistribution, of Dissipation: The Evidence for Choosing Among Bankruptcy Hypotheses”, 72 Wash. U.L.Q. 955 (1994); James W. BOWERS, "The Fantastic Wisconsylvania Zero-Bureaucratic Cost School of Bankruptcy Theory: A Comment", 91 Mich. L. Rev. 1773 (1993); Thomas H. JACKSON, "Bankruptcy, Nonbankruptcy Entitlements and the Creditors' Bargain”, 91 Yale L. J. 857 (1982); Robert K. RASMUSSEN \& David A. SKELL, Jr., "The Economic Analysis of Corporate Bankruptcy Law”, 3 Am. Bankr. Inst. L. Rev. 85 (1995); David A. SKEEL, Jr., "Rethinking the Line Between Corporate Law and Corporate Bankruptcy", 72 Tex. L. Rev. 471 (1994); Theodore EISENBERG \& Stefan SUNDGREN, "Is Chapter 11 Too Favorable to Debtors? Evidence From Abroad”, vol.09, no.01, Rio de Janeiro, 2016.pp. 545-585 548 
estudio de tres años por parte de una Comisión de numerosos expertos concursales estadounidenses, en diciembre de 2014 ha sido publicado el Informe del American Bankruptcy Institute, la principal entidad que nuclea a los profesionales de la insolvencia en los Estados Unidos, acerca de las propuestas de reforma del Chapter Eleven para el Poder Legislativo, documento de unas cuatrocientas páginas de extensión y con casi mil cien notas de fuentes $y$ antecedentes previos ${ }^{7}$.

El Chapter Eleven tiene por objeto regular un proceso concursal de "reorganización" de la empresa en crisis o en estado de cesación de pagos ("Reorganization Procedure") a fin de sanearla económica o financieramente, solucionar el problema económico o financiero, facilitar su continuación empresarial y así librarla de su liquidación judicial forzosa, solución final reglada en el Chapter Seven de ese Código Concursal. Excepcionalmente el Chapter Eleven prevé casos de liquidaciones de bienes parciales o totales en condiciones más ventajosas que los otros procedimientos liquidatorios en el derecho de los Estados Unidos.

Este trabajo constituye una comparación entre los procedimientos establecidos por el Chapter Eleven del United States Code y las normas referidas a los procedimientos colectivos extrajudiciales, (usualmente denominados "Out of Court Restructurings" o simplemente "Workouts", entre otros) en el derecho norteamericano y aborda los temas relativos a la elección alternativa de ambos procedimientos, con sus ventajas e inconvenientes, la negociación con los principales prestamistas con preferencias, la negociación con los pequeños acreedores y la utilización de técnicas de financiación postconcursal. En este trabajo he seguido la sistemática de

82 Cornell L. Rev. 1532 (1997); Linda J. RUSCH, "Bankruptcy Reorganization Jurisprudence: Matters of Belief, Faith and Hope Stepping into the Fourth Dimension”, 55 Mont. L. Rev. 9 (1994); Edward A. ALTMAN, "Evaluating the Chapter 11 Bankruptcy Reorganization Process", 1 Colum. Bus. L. Rev. (1993); Lawrence A. WEISS, "Bankruptcy Resolution: Direct Costs and Violation of Priority of Claims”, 27 J. Fin. Econ. 285 (1990); Lynn M. LOPUCKI, “The Trouble With Chapter 11”, 1993 Wis. L. Rev. 729. Una revision útil del debate y las críticas para propuestas de reforma puede verse David A. SKEEL, Jr., "Markets, Courts and the Brave New World of Bankruptcy Theory”, 1993 Wis. L. Rev. 465.

${ }^{6}$ Cfr., entre otros, Donald R. KOROBKIN, "Contractarianism and the Normative Foundations of Bankruptcy Law", 71 Tex. L. Rev. 541 (1993); Lynn LOPUCKI, "Strange Visions in a Strange World: A Reply to Professors Bradley and Rosenzweig”, 91 Mich. L. Rev. 79 (1992); Lawrence PONOROFF, "Enlarging the Bargaining Table: Some Implications of the Corporate Stakeholder Model for Federal Bankruptcy Proceedings”, 23 Cap. U.L. Rev. 441 (1994); Donald R. KOROBKIN, "Rehabilitating Values: A Jurisprudence of Bankruptcy”, 91 Colum. L. Rev. 717 (1991); John D. AYER, "Through Chapter 11 With Gun or Camera, But Probably Not Both: A Field Guide”, 72 Wash U.L.Q. 883 (1994); Hon. Frank H. EASTERBROOK, "Is Corporate Bankruptcy Efficient?”, 27 J. Fin. Econ. 411 (1990); John D. AYER, "Bankruptcy as an Essentially Contested Concept: The Case of the OneAsset Case”, 44 S.C.L. Rev. 863 (1993); Jean BRAUCHER, “Bankruptcy Reorganization and Economic Development”, 23 Cap. U.L. Rev. 499 (1994); David Gray CARLSON, "Bankruptcy Theory and the Creditors' Bargain”, 61 U. Cin. L. Rev. 453 (1992); Christopher W, FROST, "Bankruptcy Redistributive Policies and the Limits of the Judicial Process", 74 N. C. L. Rev. 75 (1995); Donald R. KOROBKIN, "The Unwarranted Case Against Corporate Reorganization: A Reply to Professors Bradley and Rosenzweig”, 78 Iowa L. Rev. 669 (1993); Elisabeth WARREN, “Bankruptcy Policymaking in an Imperfect World”, 92 Mich. L. Rev. 336 (1993); Elisabeth WARREN, "The Untenable Case for the Repeal of Chapter 11”, 102 Yale L.J. 437 (1992); Elisabeth WARREN, “Bankruptcy Policy”, 54 U. Chi. L. Rev. 775 (1987); William C. WHITFORD, ”What’s Right About Chapter 11 ”, 72 Wash. U.L.Q. 1379 (1994).

7 Cfr. American Bankruptcy Institute Comission to Study the Reform of Chapter 11: 2012-2014 Final Report and Recommendations. El informe original y completo puede encontrarse en la página web https://abiworld.app.box.eom/s/wircv5xv83aavl4dp4h . 
los autores norteamericanos respecto de los tópicos tratados y en el modo de hacerlo, lo que tiene importantes diferencias sistemáticas comparadas con otros países del sistema continental europeo.

Los procesos concursales norteamericanos de reestructuración tanto de la empresa en cuanto organización económica como de su pasivo han cambiado drásticamente con la reforma general de 1978 del United States Bankruptcy Code, norma federal que regula uniformemente la materia concursal en todo el país. Hoy en día esta regulación se ha expandido de modo amplio de manera tal que es necesaria una tarea de complementación profesional en materias tan dispares como, por ejemplo, los procesos extrajudiciales, los "Prepackaged Proceedings", los “Securities", el sector bancario, los procesos judiciales activos y pasivos del deudor, los derechos reales, los impuestos, las cuestiones medioambientales, laborales y jubilatorias y, finalmente, no sólo las cuestiones nacionales o domésticas sino también las multinacionales.

Sin duda que un proceso ordenado y justo que gobierne los momentos de crisis o cesación de pagos de una empresa constituye una herramienta integral y necesaria para economías del sistema de mercado capitalista a fin de lograr no sólo la subsistencia de una empresa con posibilidades de continuar -en lugar de su automática liquidación- sino también la posibilidad de inyección o recolección de nuevos capitales para una economía cuya expectativa es lograr un crecimiento sostenido. Ello sin perjuicio de ulteriores consideraciones no meramente materialistas, sino de orden ético y, dentro del mismo, también de consideraciones sobre el bien común que se encuentra involucrado en la actividad empresarial en reorganización, como parte de la ética social.

Como surge de la jurisprudencia americana la amplitud de la situaciones de crisis incluye casos de empresas saludables que tienen una abultada deuda financiera de corto y mediano plazo, hasta negocios que deben ser drásticamente reestructurados, reorganizados o diversificados o bien empresas que súbitamente se han visto envueltas en deudas originadas por responsabilidades patrimoniales derivadas de demandas colectivas tales como por ejemplo las originadas por sustancias cancerígenas, dispositivos intrauterinos, tabaco o drogas. Incluso los objetivos del legislador y de la jurisprudencia incluyen la promoción de la evitación del acogimiento de la empresa a las normas concursales de este Chapter Eleven en beneficio de lograr un arreglo extrajudicial de la crisis, ya que las partes interesadas están en condiciones de juzgar cuál de los dos procedimientos les es más beneficioso y en la mayoría de los casos ellas son reacias a acudir a los tribunales.

La tendencia al individualismo propio del sistema económico estadounidense, sin embargo, ha finalizado por rechazar la idea -en sede principalmente del poder legislativo nacional- de que el objetivo del Chapter Eleven es lograr preponderantemente la liquidación de una empresa en 90 o 180 días, principalmente por razones como, por ejemplo, la dificultad para acceder a préstamos de capital, su creciente costo financiero, los limitados costos causídicos del proceso en comparación con otros procesos y la eficacia de la actividad de los comités de acreedores que ahorran grandes costos de negociación realizada por profesionales. Por el contrario, las razones 
económicas para apoyar las normas e instituciones del Chapter Eleven han prevalecido frente a los costos y gastos del proceso, evitando por otra parte innecesarias liquidaciones en detrimento patrimonial de los interesados.

Algunas de las instituciones legisladas en el Chapter Eleven han sido adoptadas con modificaciones por algunos países europeos, americanos y asiáticos, por lo que puede decirse que han constituido ejemplos para la regulación de nuevos problemas de la dinámica concursal. Esto ha sido constatado en las nuevas normas concursales sancionadas en los últimos años por España, Italia, Alemania, China, Chile y Brasil, entre otros. Uno de esos ejemplos es, por ejemplo, el instituto del Cramdown en el sistema concursal argentino.

En su caso también haré referencia tangencial a los distintos ordenamientos locales de los Estados de Estados Unidos que regulan sistemas de cobro, negociación y reestructuración de deuda, y que conviven con el sistema concursal federal de este país.

\section{SOBRE LA ELECCIÓN ALTERNATIVA ENTRE EL PROCESO DEL CHAPTER ELEVEN Y LOS PROCEDIMIENTOS EXTRAJUDICIALES EN ESTADOS UNIDOS.}

Frente a la crisis o estado de cesación de pagos, la empresa tiene dos alternativas: la liquidación de sus bienes y consecuente satisfacción -en la mayoría de los casos en una pequeña proporción de las deudas- de los acreedores con su producido, o bien la superación de la crisis o cesación de pagos reestructurando su estructura empresarial, reestructurando su pasivo o reestructurando ambos. Para alcanzar la segunda alternativa las partes prefieren acudir a técnicas de negociación en procedimientos informales extrajudiciales conocidos como "Consensual Restructurings", "Out-of-Court restructurings", o también "Workouts", ”Extensions", "Compositions" y, en el caso de que fracasen, acuden a la alternativa del proceso judicial formal regulado en el Chapter Eleven del United States Bankruptcy Code, norma federal que regula la materia concursal.

Dentro de los procedimientos no liquidativos los operadores jurídicos consideran como solución satisfactoria preeminentemente a los arreglos extrajudiciales como naturales y más convenientes y sólo excepcional o subsidiariamente a los procesos judiciales preventivos de la quiebra, conforme surge de la jurisprudencia analizada y la experiencia alcanzada por los Estudios Jurídicos de abogados especializados en la materia concursal. Debe aquí destacarse que en los Estados Unidos las partes interesadas adjudican una marcada preponderancia a los sistemas extrajudiciales previos y preventivos de los judiciales a fin de solucionar un conflicto, lo que también se verifica en la materia concursal.

El "Consencual Out-of-Court Restructuring" o "Workout" puede ser definido como un proceso no judicial a través del cual la empresa que se encuentra en dificultades financieras, arriba a un acuerdo con sus acreedores más significativos para ajustar las obligaciones de la misma. Dado que la determinación de las causas de la crisis y sus posibles soluciones lleva tiempo, las reestructuraciones consensuales son típicamente un proceso lento (para los estándares de los plazos estadounidenses, que son más cortos que los frecuentemente se verifican vol.09, nº. 01, Rio de Janeiro, 2016. pp. 545-585 
en otros países). Frecuentemente, la apreciación del problema por los directores de la empresa es contrario o contradictorio con la visión de los acreedores participantes en la negociación y por ende los segundos intentarán influir en los primeros a través de la designación de profesionales tales como contadores, abogados y otros consultores, todo a cargo de la empresa. Sobre cuáles acreedores participarán ello dependerá de la naturaleza del problema económico o financiero, pero para lograr el éxito del arreglo se requiere la participación de los principales prestamistas, los mayores proveedores $y$, dependiendo de las circunstancias, otras organizaciones o entidades tales como los sindicatos y organismos gubernamentales. La orquestación de la negociación puede sufrir demoras. En muchos casos el Directorio o la dirección empresarial pueden dudar antes de negociar, a fin de elaborar y testear su propia propuesta de reestructuración. Frecuentemente los directores piensan que si la primera propuesta es justa será aceptada por los acreedores, pero en la realidad la "primera oferta" suele ser considerada por los acreedores sólo como tal, es decir, una propuesta inicial para comenzar la negociación. Por lo general se considera que el arreglo extrajudicial es más beneficioso para la empresa que las posibilidades que ofrece el Chapter Eleven judicial, más allá de las dificultades que deben ser superadas para arribar al mismo ${ }^{8}$.

Estadísticamente se ha demostrado que aproximadamente la mitad de todas las reestructuraciones empresariales son producto de un arreglo o composición extrajudicial ${ }^{9}$.

\section{PREPACKAGED CHAPTER ELEVEN}

Como parte de las alternativas extrajudiciales cabe mencionar las instituciones que giran alrededor del denominado "Prepackaged Chapter Eleven". Tomada de la jerga propia de las comidas rápidas preparadas con anticipación a la venta, en esta materia se denomina "Pre-Packaged Plan" a aquél que es negociado y acordado por los empresarios y los abogados especializados en concursos con anticipación al comienzo formal del proceso del Chapter Eleven, con frecuencia antes de que la crisis tome estado público. Este es un acuerdo extrajudicial logrado entre el deudor y los principales financistas y algunos acreedores pero que necesita la aprobación judicial a fin de asegurarse la virtual aceptación del resto de los acreedores a sumarse al arreglo, ya que no fue lograda la unanimidad de la aceptación de los acreedores. Si hubiera unanimidad no sería necesario el proceso concursal. La idea básica de esta institución es que mucho de la negociación que se da en el Chapter Eleven puede realizarse exitosamente antes del comienzo del proceso concursal ${ }^{10}$.

\footnotetext{
${ }^{8}$ Cfr. AAVV. WEIL, GOTSHAL \& MANGES, LLP, Reorganizing Failing Businesses, A Comprehensive Review and Analysis of Financial Restructuring and Business Reorganization, Rev. Edition, American Bar Association, Chicago, 2006, ps. 1-2 (A continuación será citada como WEIL, seguida del Volúmen correspondiente, el número del capítulo y la página correspondiente). ${ }^{9}$ Cfr. Stuart C. Gilson et al., Troubled Debt Restructurings: An Empirical Study of Private Reorganization of Firms in Default, $27 \mathrm{~J}$. Fin. Econ. 315, 345 (1990).

${ }^{10}$ Cfr. Elizabeth WARREN, Chapter 11: Reorganizing American Businesses, Aspen Publishers, Wolters Kluwer Law \& Business, Austin / Boston / Chicago / New York / The Netherlands, 2008, ps. 164-166. Esta obra será citada en adelante como WARREN, 2008.
} 
En este período la empresa arregla la financiación postconcursal, identifica los contratos que asumirá o rechazará, negocia con los acreedores cuánta deuda podrá ser dada por cancelada o perdonada para que pueda sobrevivir la empresa y, de modo más crítico, la empresa debe elaborar un plan empresarial realista para su propia recuperación o reorganización futura de modo tal que sea creíble su continuación a los ojos de los prestamistas postconcursales. Dado que las negociaciones y acciones preconcursales están orientadas al cumplimiento de los objetivos y requerimientos de la ley concursal, los tribunales suelen revisar si ellas son legalmente adecuadas. Concordantemente la Section 1126(b) del Código de Bancarrotas establece que la votación preconcursal será ratificada si ella reúne los requisitos legales y la \$1125(a) establece que los votos así obtenidos obligarán a los acreedores y pueden ser usados en sede concursal para confirmar el plan. Para facilitar las cosas algunas regulaciones locales (como por ejemplo la de Delaware y el Distrito Sur del Estado de Nueva York) disminuyen las exigencias sobre publicidad propias del procedimiento ordinario del Chapter Eleven para este procedimiento de "Pre-Packaged Plan". La velocidad de este procedimiento puede ser muy rápida, de modo tal que puede durar treinta días o menos, solucionando de esta forma la negatividad que puede tener la publicidad de una bancarrota de una empresa, que es lo que justamente procura este proceso. No obstante esto, si bien los abogados del mismo aducen la rapidez y los bajos costos, no todos lo encuentran favorable, especialmente aquellos acreedores que no negociaron su crédito o que incluso se opusieron al plan del deudor y ven terminado el proceso antes de que comience en sede judicial ${ }^{11}$.

En apretada síntesis pueden señalarse las principales características de esta vía extrajudicial o prejudicial en estos términos ${ }^{12}$ :

1. El deudor intenta arribar a un arreglo con los principales acreedores en un marco semejante al institucionalizado en el proceso judicial del Chapter Eleven, incluyendo anticipadamente una declaración de intención destinada a obtener la conformidad de los acreedores, un ofrecimiento de pago y un plan de reorganización empresarial.

2. Cuando termina toda la actividad preliminar el deudor iniciará su proceso de Chapter Eleven junto con su plan de reorganización, sus declaraciones informativas a los terceros, las aceptaciones de los acreedores y peticionará al juez concursal la audiencia con los acreedores para discutir sobre la adecuación de sus declaraciones informativas a los terceros, la aprobación de lo actuado y la confirmación judicial de su plan de reorganización.

3. No obstante que el estatuto del Chapter Eleven no se refiere expresamente al "Prepackaged Chapter Eleven" en esos términos, los autores han encontrado su equivalente estatutario en las Sections $1125 \mathrm{y}$ 1126 del Bankruptcy Code ya que el primero se refiere a la revelación adecuada de información a

\footnotetext{
${ }^{11}$ Ibídem.

12 Cfr. Michael J. Crames, Herbert S. Edelman \& Andrew A. Kress, The Fundamentals of Bankruptcy and Corporate Reorganization, Juris Publishing, 2003, ps. 201-214.
} 
terceros antes de la solicitud de la aceptación del plan y el segundo regula el procedimiento necesario para la adecuada aceptación del plan.

4. En este estado es necesario un adecuado y profundo intercambio de información entre el deudor y los acreedores que les permita tomar decisiones con sólidos fundamentos sobre los negocios y sobre la suerte de sus créditos y de la propuesta ofrecida en el plan a fin de lograr su aprobación.

5. Para que la revelación de la información sea adecuada al objetivo de la institución los tribunales han brindado una lista de factores a evaluar, a saber: descripción de los activos disponibles y su valuación, información financiera que permita a los acreedores evaluar la propuesta y decidir la conformidad, análisis del caso de liquidación y una descripción del plan.

6. Durante el acuerdo negociado entre el deudor y los acreedores las partes acuerdan una reunión, junta o asamblea con los principales acreedores de diversas clases que se verán afectados por la reestructuración de la deuda. En esta reunión se forman comités informales o no oficiales con los representantes de los acreedores seleccionados que negociarán el acuerdo sobre el plan. Si no se cumple con las provisiones de la Section 1126 del Bankruptcy Code el Prepackaged plan se verá perjudicado.

7. El plan debe reunir todos los requerimientos del Bankruptcy Code y el deudor debe seguir las restantes normas aplicables.

8. Un caso fallado en 1991 ha proporcionado un plexo normativo rico en un caso de una empresa de envergadura nacional ${ }^{13}$, que condujo una transacción financiera de "Leveraged Buyout" (LBO) que englobó temas como una venta de capital accionarial, un proceso de "Due Dilligence" llevado a cabo por un comité de tenedores de deuda, una propuesta de plan de reorganización empresarial y la legalidad de la votación del plan.

9. Una de las notables ventajas del "Pre-Packaged Plan" es su menor duración en el tiempo comparado con el proceso del Chapter Eleven. Es común que el primero tome entre 30 y 120 días, mientras que el segundo suele durar al menos un año y frecuentemente entre dos y tres años. Asimismo los honorarios y gastos son notoriamente menores en el primero respecto del segundo, ya que son necesarias muchas más tareas profesionales jurídicas y económicas.

10. El comienzo del proceso del Chapter Eleven puede amenazar el éxito económico de la empresa, dependiendo éste del rubro y envergadura de la misma. Frecuentemente ese proceso acarrea una pérdida de confianza de los terceros en el futuro de la empresa, especialmente de los acreedores y clientes y la emigración o dificultad para contratar personal clave en el desarrollo empresarial (especialmente gerentes y directores de la empresa), como ocurre en las empresas de servicios, agencias de publicidad, empresas de logística y distribución, agencias de viajes, etc. El estigma concursal tiene su efecto traducido

${ }^{13}$ Cfr. In Re Southland Corp., 124 B.R. 211 (Bankr. N. D. Tex. 1991), especialmente las págs. 213 y ss. vol.09, nº. 01, Rio de Janeiro, 2016.pp. 545-585 
en una mayor dificultad de las relaciones económicas de la empresa, a veces sólo por el mero hecho de haberse concursado.

11. Dentro del proceso del Chapter Eleven a veces se han dado "Hostiles Takes Overs" ("Tomas de control hostiles") que han culminado con el cambio de dueño de la empresa deudora, como ocurrió en el caso de Macy's, una gran tienda minorista, en el que un tercero pagó más que lo que el deudor podía ofrecer a cambio de asumir la titularidad de la empresa. Esto ocurrió luego de que fuera rechazado el plan propuesto por el deudor. Este riesgo de cambio de titularidad de la empresa de modo no voluntario no ocurre en el Prepackaged Chapter Eleven.

12. En este proceso el deudor tiene mayor libertad de movimientos, ya que el Directorio conserva plenamente sus facultades de gobierno y administración comercial (al igual que en el Chapter Eleven), pero no está sometido a la vigilancia, supervisión y monitoreo pormenorizados de los distintos comités de acreedores y del propio juez concursal, que debe autorizar los actos que no sean propios del curso ordinario de los negocios, como ocurre en el Chapter Eleven.

13. El peligro o dificultad para el éxito del Prepackaged Chapter Eleven radica en los siguientes hechos o situaciones, a saber:

a. Identificación de las partes interesadas a las que se debe notificar las dificultades económicas de la empresa y negociar las condiciones futuras de la financiación y pago.

b. Suspensión del crédito por parte de los acreedores. En algunos casos de procesos de Chapter Eleven los deudores han obtenido - de modo claramente excepcional- una "First Day Order" (medidas inmediatas o preliminares emitidas en los primeros días del concursamiento) consistentes en la autorización de la continuación del pago de determinadas deudas anteriores al concurso a fin de posibilitar la continuación de las operaciones empresariales, por un corto plazo. Estas medidas son excepcionales y limitadas a proveedores esenciales para la continuación empresarial y muchas veces habiéndose logrado un compromiso de continuación de la provisión del bien o del servicio al deudor durante el proceso del Chapter Eleven ${ }^{14}$. Estas medidas son propias del Chapter Eleven pero en los Prepackaged en algunas oportunidades el deudor negoció con estos determinados acreedores esenciales con éxito.

c. Posibilidad de algún acreedor de obtener una apertura concursal involuntaria del deudor, conforme la Section 303 del Bankruptcy Code, a fin de detener el transcurso del tiempo para evitar o recuperar bienes enajenados por transferencias con preferencias o fraudulentas (conforme las Sections 547 y 548 del Bankruptcy Code); o bien colocar al deudor bajo supervisión judicial en casos de sospecha de actos fraudulentos, mala administración o

${ }^{14}$ Cfr., por ejemplo, In re FF Holdings Corporation, Case n. 98 -37 (Bankr. Del. January 7, 1998) (Farnin C.J.). vol.09, nº. 01, Rio de Janeiro, 2016.pp. 545-585 
desatención de los acreedores; o, finalmente, impedir al deudor la implementación de un arreglo extrajudicial perjudicial o no razonable para todos los acreedores. No obstante que el proceso concursal involuntario provocará el comienzo prematuro de la faz concursal judicial, ello no destruye la posibilidad del deudor de reorganizarse antes de la liquidación definitiva. Por ejemplo un proceso involuntario conforme el Chapter Seven (de liquidación) puede convertirse automáticamente en un proceso concursal de Chapter Eleven (de reorganización) conforme la Section 706 del Code. Las desventajas de este concursamiento son la desaparición del Prepackaged como una alternativa, la elección del tribunal competente por los accionantes, y la sorpresa para el deudor, que puede estar desprevenido respecto de la preparación de sus empleados, proveedores y clientes para el evento.

\section{COMPOSITIONS, EXTENSIONS Y WORKOUTS}

Conforme Ferriel y Janger ${ }^{15}$, los denominados "Compositions" y "Workouts" son métodos extrajudiciales de negociación y arreglo de deudas entre el deudor y sus acreedores que tiene sus raíces en el common law contractual. El término "Workout" designa un acuerdo entre el deudor y un acreedor, o más propiamente, entre el deudor y un grupo de acreedores. El término "Composition" se refiere a un acuerdo entre el deudor y la totalidad de sus acreedores o al menos a la totalidad de los acreedores más significativos, cuyo contenido está dado por una quita en el monto de la deuda o un aplazamiento de las obligaciones con plazos más extendidos en el tiempo. No obstante esta aproximación a una definición, esta terminología no tiene un sentido legislativamente unívoco y suelen usarse sin mayores precisiones. Lo cierto de estas expresiones que designan la institución, no es la precisión terminológica sino su substancia legal y su sentido práctico. La problemática de ambos se refiere a dos elementos: la llamada "“Consideration"” contractual entre las partes, (es decir, la referencia a las contraprestaciones del acuerdo) y a los efectos frente a terceros (que no participaron en el acuerdo). Si el acreedor acepta una quita o espera en la deuda para el futuro y se obliga a continuar proveyendo sus bienes o servicios al deudor y éste sólo se limita a cumplir una parte de sus obligaciones preexistentes, en tales condiciones puede no existir una suficiente "“Consideration"” en el contrato de acuerdo entre las partes, por lo que el mismo puede devenir inejecutable conforme la norma del Uniform Commercial Code, Section 2-209 (2002). Para que esto no ocurra el deudor debería mejorar su posición, por ejemplo ofreciendo una nueva garantía inmobiliaria o mobiliaria a tal acreedor, pero esta transferencia puede ser impugnada en sede concursal si fue realizada en el período de tiempo de 90 días anteriores al comienzo del proceso concursal. Para solucionar esto, en muchos

${ }^{15}$ Cfr. Jeff Ferriell y Edward J. Janger, Understanding Bankruptcy, Matthew Bender \& Co., Inc. (Lexis Nexis Group), 2007, ps. 9496. Para este tema también puede consultarse Winton E. Williams, Resolving the Creditor's Dilemma: An Elementary Game. Theoretic Analysis of the Causes and Cures of Counterproductive Practices in the Collection of Consumer Debt, 48 Fla. L. Rev. 607 (1996). 
"Workouts" las partes acuerdan no liberar al deudor de todas sus obligaciones hasta 90 días después del último pago conforme el acuerdo.

Una "Composition" multilateral entre el deudor y muchos de sus acreedores es más complicado, no sólo por el número de éstos sino también ya sea por tener o bien por carecer de garantías reales sobre bienes del deudor, lo que puede generar desconfianza hacia él y entre los mismos acreedores. Cuando los acreedores arriban a acuerdos entre ellos aceptando quitas o esperas en pro de mejorar la posición del deudor común no existe el mencionado problema respecto de la "“Consideration"”. Pero el principal problema es lograr arribar a un acuerdo con la totalidad de los acreedores, condición para que el acuerdo sea ejecutable. Cualquier acreedor rebelde o recalcitrante puede echar por tierra la negociación si exige más de lo que el deudor puede cumplir o embargar bienes esenciales para el giro comercial. Esta es la mayor debilidad del "Workout". Sin embargo, a pesar de que el Chapter Eleven prevé mecanismos para imponer una solución consensuada por la mayoría de los acreedores a los acreedores resistentes y una valuación razonable de los bienes del deudor, las "Compositions" ofrecen algunas ventajas respecto del mismo, entre los que cabe mencionar los menores costos para el deudor, la no exposición pública a terceros de la situación económica del deudor y el no sometimiento del deudor al escrutinio y control judicial. Las "Compositions" y el proceso del Chapter Eleven no se excluyen mutuamente. En los casos de los "Prepackaged Chapter Eleven" el deudor que consigue las mayorías necesarias de acreedores aceptantes del acuerdo en los términos de la Section 1126 del Bankruptcy Code puede presentar luego el plan aceptado en sede judicial antes del comienzo del juicio ante el juez y ante los restantes acreedores para su confirmación judicial, que, en tales condiciones, es más fácil que sea aprobado ${ }^{16}$.

Numerosos deudores han solucionado sus problemas económicos o financieros mediante los Workouts, en una proporción cercana al cincuenta por ciento de todas las reorganizaciones empresariales en los Estados Unidos ${ }^{17}$. Autores como Tabb engloban terminológicamente al "Workout" como el género conceptual de arreglo contractual privado extrajudicial y a otros acuerdos semejantes como la "Composition" y la "Extension" como especies del mismo. Este autor describe la "Composition" como un contrato entre el deudor y dos o más acreedores al que adhieren otros acreedores participantes en la negociación, por el cual aceptan un pago menor al total de sus créditos. Una "Extension" es un contrato entre el deudor y al menos dos o más acreedores al que adhieren otros acreedores por el cual se le concede una extensión en el tiempo o espera en el repago de los créditos. Ambos institutos pueden combinarse y entonces constituyen una quita y una espera simultáneamente ${ }^{18}$.

\footnotetext{
${ }^{16}$ Ibídem. Sobre esto último véase Ronald Barliant, Dimitri G. Karcazes \& Anne M. Sherry, From Free-Fall to Free-For-All: The Rise of the Pre-Packaged Asbestos Bankruptcies, 12 Am. Bankr. Inst. L. Rev. 441 (2004) y la doctrina del caso United Artists Theatre Co. v. Walton, 315 F. 3d 217, 224 n. 5 (3d Cir. 2003).

${ }^{17}$ Cfr. el estudio de Stuart C. Gilson et al., Troubled Debt Restructurings: An Empirical Study of Private Reorganization of Firms in Default, 27 J. Fin. Econ. 315, 345 (1990).

${ }^{18}$ Cfr. Charles Jordan Tabb, The Law of Bankruptcy, The Foundation Press, Inc., Westbury, New York, 1997, ps. 25-27. vol.09, nº. 01, Rio de Janeiro, 2016. pp. 545-585 
Este acuerdo es oponible a todas las partes y produce un "Discharge" de todas las obligaciones, sin que esto contradiga la regla del caso inglés Foakes v. Beer ${ }^{19}$ en virtud del cual se estableció que la necesaria “"Consideration"” que fundamenta el "Discharge" de una obligación no existe cuando el acreedor acepta un pago menor que el total de la deuda no disputada y líquida. Esto es así por una parte por haberse pactado con al menos dos acreedores y por otra parte, la "Consideration" de este contrato consiste en las mutuas promesas de aceptación de un importe menor que el total de la deuda y la renuncia o abstención de ulteriores gestiones extrajudiciales o judiciales de cobro de la misma contra el deudor. No obstante esto, a mayor abundamiento, algunos Estados de Estados Unidos han rechazado en el ámbito contractual de los "Workouts" la lisa y llana aplicación de la regla del caso inglés de 1884 y han aceptado que incluso con la participación de un solo acreedor el "Workout" es ejecutable ${ }^{20}$. El acuerdo puede verse amenazado en su cumplimiento en caso de que un elevado número de acreedores que no lo hayan aceptado agredan patrimonialmente al deudor, especialmente cuando el deudor haya arreglado de modo desigual (es decir de modo distinto a acreedores del mismo rango o categoría) con algunos de los acreedores aceptantes del acuerdo. A fin de evitar estos problemas los acreedores pueden incluir una cláusula que exija un porcentaje mínimo de acreedores participantes. Una cuestión importante es qué ocurre con los derechos de los acreedores si el deudor incurre en cesación de pagos antes de haber cumplido totalmente con lo acordado. Se plantea la existencia o no de la novación de las obligaciones y si los acreedores pueden ejecutar los bienes del deudor por los montos originarios o no ${ }^{21}$. Algunos autores afirman que las condiciones obligacionales originarias pueden revivir, mientras que, en cambio, en sede judicial y conforme el Chapter Eleven, las deudas han quedado novadas totalmente ("Discharged") en cuanto el plan es aprobado ${ }^{22}$. El principio es que el acuerdo no es oponible a los acreedores que no participaron en el acuerdo y pueden ejecutar los bienes del deudor por la totalidad de la deuda. Esta es una de las debilidades del "Workout" y de ahí la necesidad del proceso del Chapter Eleven a fin de oponer el acuerdo a los acreedores disidentes conforme la Section 1131(d) del Code. Sin duda esto será un arma de negociación del deudor y motivará a los acreedores a aceptar las condiciones ofrecidas en el acuerdo extrajudicial. Otra cuestión que puede presentarse es el otorgamiento de especiales garantías sobre todo el patrimonio del deudor a los acreedores aceptantes, no dejando nada para los no participantes ${ }^{23}$.

Las "Compositions" y las "Extensions" son remedios Estatales o locales, es decir, no federales, que suelen ser utilizados en un contexto de insolvencia, pero hoy lo son en menor medida que en el pasado. La consecuencia sustancial de un acuerdo cumplido es la liberación del deudor de la obligación de realizar ulteriores pagos. La

\footnotetext{
${ }^{19}$ Cfr. Foakes v. Beer, L.R. 9 A.C. 605 (H.L. 1884), de la House of Lords.

${ }^{20}$ Cfr. E. Allan Farnsworth, Contracts, $\$ 4.25$, at 308 (2d ed. 1990).

${ }^{21}$ Cfr. Charles Jordan Tabb, op. cit., p. 26.

${ }^{22}$ Cfr. Elizabeth Warren / Jay Lawrence Westbrook, The Law of Debtors and Creditors, Text, Cases and Problems, 6th Ed., Wolters Kluwer, Austin, Boston, Chicago, New York, The Netherlands, 2009, p. 94.

${ }^{23}$ Ibídem.
} 
"Composition" puede ser invalidada si el deudor ha inducido a error mediante fraude, ha preferido a ciertos acreedores en desmedro de los restantes o se ha involucrado en otros tipos de conducta desviada. En este contexto el Chapter Eleven brinda mayores posibilidades de alcanzar las finalidades concursales y posee mayores ventajas que los remedios locales. Sin embargo, el proceso concursal es más oneroso y lento. Si los especiales poderes y controles del juez concursal y de los comités de acreedores no son necesarios (por ejemplo porque los bienes y obligaciones del deudor son pequeños y los acreedores están de acuerdo) puede ser más conveniente el uso de los remedios locales, que son más silenciosos y pacíficos que el proceso concursal y en muchos casos resultan de crítica importancia para la continuidad de las operaciones negociales del deudor. Sin embargo, esta quietud y la posibilidad de los insiders de manipular estos remedios proveen menos defensas contra comportamientos fraudulentos relacionados con el acuerdo, lo que es quizá la razón por la cual ha disminuido su uso en las últimas décadas. A ello se agrega que son más o menos populares según de qué Estado se trate ${ }^{24}$.

\section{OTROS REMEDIOS COLECTIVOS ESTATALES}

Además del Chapter Eleven, los "Prepackaged Chapter Eleven”, "Pre-Packaged Plan”, "Compositions”, "Extensions" y "Workouts", existen otros procedimientos colectivos estatales no federales de cobro de deudas y negociación de arreglos de forma paralela a las normas federales, que en cada Estado reúnen características y efectos diferentes entre sí y que son dignos de mención. Tales son los "Assignment for the Benefit of Creditors" (usualmente llamados "ABC") y los "Receiverships". El ABC no tiene el efecto de operar la "Discharge" de las deudas negociadas con los acreedores, atento la supremacía que tienen las normas concursales federales del Chapter Eleven respecto de la novación de las deudas concursales ${ }^{25}$ y puede producir un "blindaje" patrimonial a fin de evitar posteriores medidas precautorias y preferencias sobre los bienes del deudor atento que entran bajo la custodia del tribunal ("Custodia Legis"). En algunos Estados pueden otorgarse preferencias a determinados grupos de acreedores mientras que en otros Estados esto está prohibido ${ }^{26}$.

El "Receivership" constituye el sometimiento de un patrimonio bajo el control judicial de sujetos que normalmente no pueden concursarse conforme los términos del Bankruptcy Code ${ }^{27}$ (vg. una Iglesia, un comité político, ciertas organizaciones sin ánimo del lucro, un granjero). El "Receiver" nombrado por el tribunal queda a cargo de los bienes y negocios del deudor, con poderes de negociación, gobierno y con medidas de protección del patrimonio frente a terceros. A veces pueden ser establecidos por violación de estatutos regulatorios de la actividad del deudor a fin de evitar ulteriores violaciones y facilitar la investigación de la conducta del deudor. Aquì

\footnotetext{
${ }^{24}$ Ibídem.

${ }^{25}$ Cfr., por ejemplo, Straton v. New, 283 U.S. 318 (1931), de la Corte Suprema Federal.

${ }^{26}$ Cfr. Elizabeth Warren / Jay Lawrence Westbrook, The Law of Debtors and Creditors, Text, Cases and Problems, 6th Ed., Wolters Kluwer, Austin, Boston, Chicago, New York, The Netherlands, 2009, p. 93.

${ }^{27}$ Cfr. Bankruptcy Code, \$\$ 109(b), 303(a).
} 
también opera la "Custodia Legis" del tribunal. Dada la aplicabilidad de las normas federales concursales esta institución está limitada principalmente a bancos y entidades aseguradoras a fin de lograr el cumplimiento de la reglamentación institucional que los rige, ya que estos sujetos no son concursables ${ }^{28}$.

\section{VENTAJAS COMPARATIVAS DEL ACUERDO EXTRAJUDICIAL}

Sobre los beneficios del acuerdo extrajudicial comparándolos con los del Chapter Eleven cabe apuntar aquí los siguientes ${ }^{29}$ :

1. Si bien el hecho de acogerse al proceso del Chapter Eleven en los últimos años se ha visto reducido el estigma que significa el concurso, las negociaciones extrajudiciales preservan la confianza depositada en la empresa por parte de los empleados, vendedores y clientes, incluso frente a las dificultades económicas o financieras. La confianza de los terceros que contratan con la sociedad comercial es un activo intangible importante para la empresa.

2. Las negociaciones extrajudiciales son, por otra parte, indicativas de la confianza de los acreedores en el futuro de la empresa que no acude a los tribunales para pedir ayuda. Incluso podría pensarse que en este caso la confianza de la propia empresa en sí misma es mayor que en el proceso judicial, en la que interviene la decisión del juez, ajeno a la empresa.

3. Las operaciones empresariales, principalmente las contractuales, no se ven sometidas a publicidad frente a terceros, característica propia del proceso judicial, lo que puede afectar la credibilidad de terceros en la aptitud de continuar sus operaciones comerciales. En este sentido cabe destacar que la empresa está obligada en sede judicial a revelar sus activos, responsabilidades, reclamos actuales vigentes, "executory contracts", tenedores de bonos o "securities", clientes, prestamistas, proveedores, compradores, estructura financiera, etc., cuya documentación puede ser examinada por cualquier tercero, lo que no ocurre en las negociaciones extrajudiciales. Si bien se puede obtener protección frente a la revelación de algunos tipos de información, las actuaciones concursales son públicas ${ }^{30}$. Asimismo, esta publicidad puede dar lugar a procesos contradictorios en el marco del Chapter Eleven, entre las partes (el deudor y acreedores, vendedores, clientes, etc.), lo que puede tener un efecto negativo en el desarrollo ulterior de los negocios de la empresa.

\footnotetext{
${ }^{28}$ Cfr. Elizabeth Warren / Jay Lawrence Westbrook, The Law of Debtors and Creditors, Text, Cases and Problems, 6th Ed., Wolters Kluwer, Austin, Boston, Chicago, New York, The Netherlands, 2009, p. 95.

${ }^{29}$ Cfr. WEIL, Vol. I, 1-3 / 1-7.

${ }^{30}$ Sobre esta norma puede confrontarse, por ejemplo, 11 U.S.C. $\$ 521$ (a)(1) y $\$ 704$ (8). Sobre información que debe presentar la empresa puede verse, por ejemplo, Fed. R. Bankr. P. 2004. La Exchange Act en algunos casos exige la revelación de información específica sobre "securities".
} 
4. No obstante lo anterior, para muchas empresas esa publicidad no es un grave problema, sino que es gravoso la obligatoriedad de los directores de realizar declaraciones bajo juramento ante terceros, especialmente acreedores, como ocurre en sede judicial ${ }^{31}$.

5. En contraste, en los procedimientos extrajudiciales el deudor puede revelar información empresarial limitándose a lo esencial y puede requerir confidencialidad respecto de la misma al tercero que la conoce. Cabe destacar que la confidencialidad en países como Estados Unidos no sólo es valorada sino también altamente respetada.

6. Si bien la negociación extrajudicial puede llegar a ser lenta, es más corta en el tiempo que lo que suele tardar el lograr una confirmación del plan propuesto por el deudor en el Chapter Eleven.

7. En la negociación extrajudicial el deudor puede limitarse a tratar con los acreedores que juzgue necesarios para la reestructuración, lo que flexibiliza y simplifica los procedimientos necesarios para alcanzarla. Por el contrario, el Chapter Eleven somete a todas las partes involucradas a un proceso supervisado por el juez concursal sometido a un conjunto de reglas, entre las cuales se encuentra la obligatoriedad de aprobar judicialmente las medidas empresariales extraordinarias decididas por el deudor y de aprobar la negociación y la aceptación de propuestas formuladas por el deudor, lo que puede dar lugar a incidencias contradictorias y actividad probatoria.

8. La complejidad en el Chapter Eleven es mayor por cuanto se debe dar intervención en algunas oportunidades no sólo a las partes intervinientes sino también a comités de acreedores institucionales que los representan en el juicio ${ }^{32}$, así como a otros terceros, como por ejemplo sindicatos, entes estatales de aplicación normativa, etc. Esto puede resultar por una parte en un incremento del tiempo y esfuerzo necesarios para la negociación de la suerte de la empresa y por otra parte a un incremento de la agresividad procesal, especialmente de los pequeños acreedores y de los tenedores de títulos o "securities". Por esto la negociación extrajudicial es más expeditiva que el proceso del Chapter Eleven.

9. Si bien en ambos casos el resultado de las negociaciones y de la actuación judicial no está asegurado, los costos de transacción del proceso del Chapter Eleven aumentan y son superiores a los de la negociación extrajudicial por cuanto el deudor debe hacerse cargo de los honorarios de los profesionales (abogados, contadores, bancos de inversión y otros) designados por él mismo y por los comités de acreedores y otros comités que pueden formarse; y la duración de su actuación es superior.

\footnotetext{
${ }^{31}$ Cfr., 11 U.S.C. $\$ 341$.

${ }^{32}$ Cfr. 11 U.S.C. $\$ 1102,1103$.
} 


\section{CONSIDERACIONES RESPECTO DEL MANAGEMENT DE LA EMPRESA}

En el Workout, la actividad del directorio se centra en la preparación de un plan viable de reestructuración y de un plan de negocios y documentación de soporte del mismo que deberán ser presentados a los acreedores. Comparativamente, en el Chapter Eleven a esas tareas se añaden otras a los directores de la empresa, cuyo rol se hace más ambiguo. Entre tales tareas pueden mencionarse las siguientes, a saber ${ }^{33}$ :

1. Dedicarse a mejorar o reestructurar el plan de negocios comerciales de la empresa.

2. Instrumentar la ingeniería financiera propia de la situación de la misma.

3. Monitorear diariamente las actuaciones propias del proceso judicial.

4. Actuar expeditivamente, ya que en el Chapter Eleven, dada la necesaria aprobación judicial para las operaciones extraordinarias de la empresa y los eventuales procedimientos contradictorios con las otras partes integrantes del mismo, ello dilatará en el tiempo la ejecución de las decisiones empresariales, lo que puede hacer perder muchas oportunidades de negocios. Lo mismo cabe decir acerca del emprendimiento de nuevos negocios y de exigencias propias de la competencia empresarial en el mercado.

5. La posición de los directores en el caso del Chapter Eleven asume otros deberes fiduciarios para con los acreedores, añadidos a los otros deberes fiduciarios cuando la sociedad está "in bonis", especialmente en materia de "preferencias anulables" ("Voidable Preferences") y de "transferencias fraudulentas" ("Fraudulent Conveyances"), en virtud de los cuales pueden verse obligados a ir contra los intereses de partes constituyentes de la empresa a fin de recuperar créditos previamente otorgados ${ }^{34}$.

6. El directorio se verá menos inclinado a proteger a los tenedores de capital ("Equity Securityholders") en el Chapter Eleven, en comparación con los Workouts, ya que el código expresamente establece la subordinación de esos tenedores a la suerte de los restantes acreedores ${ }^{35}$ y de hecho en muchos casos no perciben sus créditos en la distribución final de esos procesos judiciales. Asimismo el directorio puede velar más por estos tenedores de títulos en los casos de los Workouts porque de algún modo se extienden sus deberes fiduciarios hacia los accionistas.

7. La necesidad de lograr un consenso entre los acreedores es un objetivo final e insalvable de toda la negociación, ya se trate del Workout o del proceso judicial. El hecho de quedar subordinados los derechos de los acreedores al poder del juez concursal y bajo las normas del Bankruptcy Code y las

\footnotetext{
${ }^{33}$ Cfr. Weil, Vol I, 1-7 / 1-18.

${ }^{34}$ Cfr., por ejemplo lo normado en 11 U.S.C. $\$ \$ 547,548$.

${ }^{35}$ Cfr. 11 U.S.C. $\$ 1129$ (b)(2)(B). Esta regla es conocida como la "Absolute Priority Rule" y es muy importante para la negociación del plan concursal a ser confirmado por el juez.
} vol.09, nº. 01, Rio de Janeiro, 2016.pp. 545-585 
restantes normas aplicables ${ }^{36}$ limita su poder de negociación, lo que estimula la benignidad con que ellos miran las perspectivas de lograr un consenso con el deudor, favoreciendo la aceptación de sus propuestas en el Workout.

8. Como posibles causas del fracaso de la negociación extrajudicial que los directores deben tener en cuenta pueden mencionarse las siguientes, a saber:

a. la desconfianza en las perspectivas económicas de futuro de la empresa,

b. la desconfianza en la actuación de los miembros actuales del directorio,

c. la desconfianza en los términos del "business plan" de la empresa,

d. la perspectiva de recibir los acreedores un mayor valor si se inicia un proceso de Chapter Eleven o se liquida la empresa,

e. la posibilidad de obtener exitosas acciones de recomposición patrimonial mediante acciones judiciales contra transferencias fraudulentas realizadas a favor de otros acreedores,

f. la necesidad de integrar al proceso un elevado número de acreedores para lograr una exitosa reestructuración empresarial,

g. la gran complejidad de las cuestiones comprometidas en la negociación extrajudicial.

9. Respecto de las obligaciones tributarias, existen ventajas fiscales en el caso del Chapter Eleven ${ }^{37}$ que no existen en el caso de los Workouts, lo que puede desalentar llevar a cabo el segundo. Asimismo existen beneficios fiscales para casos de emisión de capital derivada de una reestructuración judicial de la empresa ${ }^{38}$ y para casos de transferencias de activos en dicho proceso judicial ${ }^{39}$.

10. Respecto de los denominados "Securities", las necesidades de financiamiento de la empresa en dificultades puede obligarla a emitirlos, dándose la situación de la simultánea aplicabilidad de las normas del Bankruptcy Code y de las de los "Securities", lo que puede ser complejo, especialmente en materia de oferta, negociación, publicidad y registración de estos títulos por el deudor concursado, como acciones integrantes del "Exchange Offer" del mismo y del "Tender Offer" ${ }^{\text {". }}$. En el 2006 se reguló especialmente la emisión de estos títulos a fin de preservar la "Fairness" de las transacciones con estos títulos ${ }^{41}$. Sobre los "Securities" existe legislación federal y estatal simultáneamente aplicable, especialmente en materia de fraude.

\footnotetext{
${ }^{36}$ Cfr., por ejemplo las limitaciones del 11 U.S.C. $\$ 1123$ (b)(1)(5).

${ }^{37}$ Cfr., por ejemplo Internal Revenue Code, $\$ 108(\mathrm{a})(1)(\mathrm{B})$.

${ }^{38}$ Cfr. por ejemplo Internal Revenue Code, $\$ 382$.

${ }^{39}$ Cfr. 11 U.S.C. $\$ 1146$ (a).

${ }^{40}$ Cfr., entre otras 15 U.S.C. $\$ 77$ y y la Securities Act $\$ 3(a)(9)$, \$4(2) y (6) y \$5 con sus respectivos incisos. Las “Tender offer” están reguladas en 15 U.S.C. $\$ \$ 78 \mathrm{~m}(\mathrm{~d})-(\mathrm{e})$ y $78 \mathrm{n}(\mathrm{d})-(\mathrm{f})$.

${ }^{41}$ Cfr. las denominadas "Going Private" Rules en 17 C.F.R. \$240.13e-3 (2006).
} 
Como puede observarse, esta sistemática no obedece a razones de lógica jurídica sino de variables del management empresarial.

\section{CASOS EN LOS QUE ES MÁS CONVENIENTE EL CHAPTER ELEVEN}

A pesar de que ordinariamente la mejor solución para la empresa en dificultades suele ser naturalmente la negociación extrajudicial, en algunos casos característicos es más conveniente la elección del proceso regulado en el Chapter Eleven a fin de lograr la reestructuración de la empresa y su pasivo. Entre estos casos típicos pueden mencionarse los siguientes, a saber ${ }^{42}$ :

1. Existencia de un gran número de juicios en contra de la empresa que dificultan notoriamente o impiden la consecución de su negocio empresarial. Un remedio importante para esta situación es el denominado "Automatic Stay" de la ley concursal ${ }^{43}$, en virtud del cual se prohíbe el inicio o continuación de las demandas judiciales contra la empresa, lo que permite al directorio focalizarse en su restructuración. Esta ventaja no existe en los Workouts. Como ejemplos de estos casos se citan Twin Laboratories y Texaco Inc. En el primero se ventiló la responsabilidad civil derivada de la producción y venta de efedra y sus productos relacionados, que causaron muertes en los consumidores y centenares de millones de dólares reclamados como indemnización en muchos diferentes Estados del país, cuyos procesos fueron consolidados en un solo Estado. En Texaco el deudor intentó protegerse de una sentencia judicial de varios miles de millones de dólares obtenida por Pennzoil Company en conexión con la adquisición de Getty Oil Company por Texaco.

2. Casos en los que se pone en tela de juicio la continuación o el reemplazo de los miembros del directorio de la empresa. El principio es que los miembros del directorio pueden continuar en el cargo, en base a la presunción conforme la cual la continuación de los mismos beneficia a la empresa ya que esos directores están familiarizados con el negocio. Consecuentemente, para reemplazar al director con un tercero, el peticionante debe demostrar que o bien ha ocurrido un caso extremo (como por ejemplo fraude, deshonestidad, incompetencia o grave negligencia en la dirección empresarial) o bien que se trata de un caso en que el reemplazo es relevante a fin de beneficiar a los acreedores, tenedores de bonos y otros interesados ${ }^{44}$.

3. Mejorar la posición financiera del deudor al obtener el "Automatic Stay" y al obtener financiamiento postconcursal, facilitado en base a que el prestamista postconcursal puede obtener un superprivilegio

\footnotetext{
${ }^{42}$ Cfr. Weil, Vol I, 1-18 / 1-21.

${ }^{43}$ Cfr. 11 U.S.C. $\$ 362(a)$.

${ }^{44}$ Cfr. 11 U.S.C. $\$ 1104$. Existe una creciente tendencia en los tribunales para la designación de un mediador en los procesos de Chapter Eleven en sus distintas etapas.
} vol.09, nº. 01, Rio de Janeiro, 2016.pp. 545-585 
sobre los activos del deudor, si se cumplen los requisitos para la "Debtor-in-Possession Financing", conforme a la ley concursal.

4. Mejorar el acceso al crédito comercial. Los acreedores preconcursales pueden verse afectados en el cobro de sus créditos, pero los acreedores postconcursales deben ser pagados en su totalidad para que el deudor pueda tener su plan confirmado judicialmente en los términos de las normas del Chapter $E_{\text {Eleven }}^{45}$. Esto puede facilitar el otorgamiento de crédito postconcursal que significará capital de trabajo para el deudor en dificultades.

5. A pesar de que en un Workout puede negociarse la finalización de ciertos contratos o la extensión de los plazos de pago, el Chapter Eleven le da a la empresa amplios poderes para aceptar, rechazar o modificar las cláusulas de los denominados "Executory Contracts" que suelen ser claves para facilitar el desenvolvimiento empresarial ulterior ${ }^{46}$, poder que no existe en el Workout.

6. Otra ventaja del Chapter Eleven que no existe en la negociación extrajudicial es el poder de reestructurar obligaciones financieras no consensualmente, es decir, imponiéndose esa solución a los acreedores disconformes, si se han cumplido algunos requisitos específicos, de acuerdo al poder del "Cramdown"47, habiendo mediado aprobación judicial sobre la aplicación de esa institución. Esa reestructuración obligacional sólo se puede conseguir extrajudicialmente mediante el acuerdo mutuo de las partes.

\section{LAS POSIBILIDADES Y VENTAJAS DE LOS PROCEDIMIENTOS PREVIOS AL CHAPTER ELEVEN}

Existen dos formas alternativas previas al Chapter Eleven que han sido calificadas por algunos autores como "el mejor de los mundos posibles" ${ }^{4}$, a saber:

El deudor puede negociar una preparación de sus acuerdos con los acreedores mediante lo que se llama un "Prepackaged Chapter Eleven" -como hemos visto supra en este trabajo- y en algunos casos esta alternativa puede constituir una solución rápida para la crisis empresarial, con ahorro de costos extrajudiciales y judiciales. En la misma el deudor negocia el plan de reorganización, prepara la declaración de su situación frente a los terceros interesados y pide los votos favorables antes del comienzo formal del procedimiento del Chapter Eleven. De este modo el deudor puede obtener los beneficios tanto de un Workout como de la reestructuración del Chapter

\footnotetext{
${ }^{45}$ Cfr. 11 U.S.C. $\$ 1129(a)(9)(A)$.

${ }^{46}$ Cfr. 11 U.S.C. $\$ 365(\mathrm{a}),(\mathrm{f}), \$ \$ 502(\mathrm{~g}),(\mathrm{b})(6)$ y (7) y $\$ 1129(\mathrm{~b})$.

${ }^{47}$ Normado en el $\$ 1129$ del Bankruptcy Code.

${ }^{48}$ Cfr. Weil, Vol I, 1-22/23.
} 


\section{Quaestio Iuris}

Eleven. Por ejemplo, en el caso Independent Wireless One Corporation (IWO) Holdings y sus afiliadas ${ }^{49}$, las partes lograron la confirmación del plan por el juez concursal 36 días luego del comienzo de las actuaciones.

Asimismo existen otras formas extrajudiciales de negociación previas al Chapter Eleven que han comenzado a utilizarse en los últimos años y han resultado exitosas como el denominado "Prenegociated Chapter Eleven", proceso en el cual el deudor inicia formalmente un Chapter Eleven y a la fecha de le revelación formal de su situación y propuesta, el deudor ya ha negociado con sus principales acreedores y ha obtenido su voto favorable al plan previamente presentado a ellos, lo que agiliza los trámites del Chapter Eleven, de ser necesario. La diferencia con el "Prepackaged Chapter Eleven" está en que el deudor no comienza la obtención de los votos favorables hasta que no haya obtenido la aprobación del tribunal de su "Disclosure Statement", esto es la declaración de su situación y propuesta a las partes interesadas. Si bien esta alternativa puede demorar más tiempo que el anterior, ha habido casos en que el tribunal ha confirmado el plan en menos de siete meses de trámite conjunto con el Chapter Eleven ${ }^{50}$.

\section{Negociación con los acreedores}

\section{Deuda con preferencias}

El deudor suele firmar contratos de financiación con entidades institucionales y bancos en los que se pactan preferencias respecto de otros acreedores. La norma la constituye cada contrato en particular. Los casos judiciales sólo registran algunos casos de responsabilidad de prestamistas en casos de daños al medio ambiente.

\section{Deuda de menor cuantía sin preferencia}

Las principales técnicas usadas por los emisores de títulos de deuda de menor cuantía (también llamados "Junk Bonds") para reestructurar los pasivos en sede extrajudicial son tres: "Tender Offers", "Exchange Offers" y "Consent Solicitations". Las partes elegirán algunas de ellas o sus posibles combinaciones de acuerdo a las circunstancias de la reestructuración del pasivo y de los objetivos buscados. Cada emisión y las respectivas recompras o rescate de títulos tiene sus propias reglas contractuales de acuerdo a cada caso, bien que con numerosas cláusulas estándar. En esta materia son aplicables no sólo normas contractuales sino también normas fiscales y sobre "Securities".

\footnotetext{
${ }^{49}$ Cfr. In re IWO Holdings, Inc., n 05-100009 (PJW) (Bankr. D. Del. Feb. 9, 2005), (orden confirmatoria del plan de reorganización).

${ }^{50}$ Cfr., por ejemplo, In re Magellan Health Serv., Inc., n0 03-40515 (PCB), (Bankr. S.D.N.Y. Oct 8, 2003). vol.09, nº. 01, Rio de Janeiro, 2016.pp. 545-585 
Respecto de los acreedores de deuda no institucional de menor cuantía es aplicable en algunos aspectos, además del propio contrato de emisión, la Trust Indenture Act de $1939^{51}$, sobre la emisión de bonos de deuda o "Debt Securities". Existen algunos elementos coercitivos en la emisión y modificación de cláusulas de estos títulos en cuyos detalles no abundaremos aquí. La jurisprudencia de Delaware ha interpretado la modificación de las cláusulas de emisión de los títulos a la luz de la buena fe contractual entre las partes ${ }^{52}$, al igual que la jurisprudencia de Nueva York, pero con una interpretación más restrictiva de la buena $\mathrm{fe}^{53}$. En algunos casos se planteó la legitimidad de permitir fusiones societarias y de permitir incurrir en un endeudamiento adicional, en el contrato del Indenture, lo que fue aceptado por los tribunales ${ }^{54}$.

\section{Utilización de tecnicas de financiación}

Conceptos como el "Structured Financing" ("financiación estructurada") o la "Asset Securitization" ("securitización de activos") se refieren a particulares técnicas de financiación del deudor concursado ${ }^{55}$. Estas técnicas de financiación postconcursal del deudor pueden proveer de liquidez al concursado con un costo menor que la financiación con garantías reales. A pesar de que estas técnicas tienen como fin individualizar y separar uno o más activos para securitizarlos e independizarlos de los riegos de la insolvencia judicial, sin duda no garantizan que ella no ocurrirá ni implicarán una indemnidad de los directores de alguna responsabilidad eventual.

El régimen de este tipo de financiación está configurado someramente de la siguiente forma ${ }^{56}$ :

1. Una típica securitización consiste en la individualización y venta de un activo por una sociedad, (denominada como "originadora") con un fin específico, hacia un ente denominado "Bankruptcyremote Vehicle" (SPV por sus siglas en inglés) y la emisión de títulos negociables comprados por el SPV, con un costo de colocación menor que otras formas de financiación tradicionales al separar ese activo del patrimonio del "originador". Entre otras ventajas, esto puede mejorar la ecuación deuda-capital propio del originador.

2. Pueden surgir conflictos sobre la calificación del contrato por las partes, que a veces puede ser considerado como una venta o bien como un contrato de financiación, lo que los tribunales han zanjado

\footnotetext{
${ }^{51}$ Cfr. Ch. 411,53 Stat. 1149 (codificada en 15 U.S.C. \$\$77aaa-77bbbb).

${ }^{52}$ Cfr. Katz v. Oak Industries, 508 A. 2d 873, 880 (Del. Ch. 1986). Ver también el caso Simons v. Cogan, 542 A. 2 d 785,787 (Del. Ch. 1987).

${ }^{53}$ Cfr. Van Gemert v. Boeing Co., 520 F. 2 d 1373 (2d Cir.), cert. denied, 423 U.S. 947 (1975) y Caplan v. Unimax Holdings, Corp., 591 N.Y.S. 2 d 28 (N.Y.App. Div. 1992).

${ }^{54}$ Cfr. Hartford Fire Insurance Co. v. Federated Department Stores, Inc. 723 F. Supp. 976 (S.D.N.Y. 1989) y Metropollitan Life Insurance Co. v. RJR Nabisco, Inc., 906 F. 2 d 884 (2d Cir. 1990).

${ }^{55}$ Puede verse al respect, como marco general: Committee on Bankruptcy and Corporate Reorganization of the Association of the Bar of the City of New York, New Developments in Structured Finance, 56 Bus. Law. 95 (2000); asimismo Committee on Bankruptcy and Corporate Reorganization of the Association of the Bar of the City of New York, Structured Financing Techniques, 50 Bus. Law. 527 (1995).

${ }^{56}$ Weil, Vol I, 4-1 / 4-22.
} 
en diversas oportunidades, sea con basamento en la prueba documental aportada por las partes contendientes, sea valorando su comportamiento $\operatorname{contractual}^{57} \mathrm{o}$, finalmente, ameritando la terminología utilizada en la contratación ${ }^{58}$.

3. La atribución de los riesgos y beneficios de la propiedad así transmitida o securitizada ha dado lugar a diversos conflictos judiciales ${ }^{59}$.

4. Pueden asimismo existir conflictos sobre las contraprestaciones en este tipo de negociaciones contractuales $^{60}$.

5. Durante cierto tiempo existieron diferencias de criterio entre las normas jurídicas y las normas contables acerca del tratamiento de este tipo de contratos, pero en los últimos años esas diferencias se han acortado. En este sentido si una transacción no era calificada como una venta conforme los Generally Accepted Accounting Principles (GAAP), con mayor razón los tribunales se resistían a considerarla jurídicamente como una venta. En cambio, en la actualidad, ambos criterios se han acercado recíprocamente gracias a los estándares contables establecidos por el Financial Accounting Standards Board (FASB) en al año 2000 para este tipo de operaciones ${ }^{61}$.

\section{Financiación y consolidación sustancial}

En este tipo de contratos financieros ha impactado la institución de la "Substantive Consolidation" (Consolidación sustantiva o sustancial), que en la actualidad todavía está en proceso de delimitación clara de sus presupuestos, límites y consecuencias, especialmente en el campo de los grupos de sociedades ${ }^{62}$. En el inicio de esa

\footnotetext{
${ }^{57}$ Cfr., por ejemplo, Major's Furniture Mart, Inc. v. Castle Credit Corp., 602 F. 2 d 538, 542-45 (3d Cir. 1979).

${ }^{58}$ Cfr., por ejemplo, Fireman's Fund ins, Coos. V. Grover (In re The Woodson Co.), 813 F. 2 d 266, 272 (9 $9^{\text {th }}$ Cir. 1987). Sobre los términos de la discusión sobre la calificación del contrato como compraventa o financiación pueden verse, entre otros: Blackford v. Commencial Credit Corp., 263 F. 2d 97, 106 (5th Cir.), cert denied, 361 U.S. 825 (1959); Rechnitzer v. Boyd (In re Executive Growth Invs., Inc.), 40 B.R. 417, 422 (Bankr. C.D. Cal. 1984); Castle Rock Indus. Bank v. S.O.A.W. Enters. (In re S.O.A.W. Enters), 32 B.R. 279 (Bankr. W.D. Tex. 1983) y West Pico Furniture Co. v. Pac. Fin. Loans, 469 P. 2 d 665 (Cal. 1970).

${ }^{59}$ Puede verse, por ejemplo, Golden Plan, 829 F. 2 d 705 y Rechnitzer v. Boyd (In re Executive Growth Invs., Inc.) 40 B.R. 417, 422 (Bankr. C.D. Cal. 1984).

${ }^{60}$ Cfr., por ejemplo, Fox v. Peck Iron \& Metal Co., 25 B.R. 674, 690 (Bankr. S.D.Cal. 1982) y Cent. Discount Co. v. Dep't of Revenue, 94 N.W.2d 805 (Mich. 1959).

${ }^{61}$ Cfr. Accounting for Transfers and Servicing of Financial Assets and Extinguishments of Liabilities, Statement of Financial Accounting Standards n 140 (Financial Accounting Standards Bd. 2000) (conocida como FAS 140).

${ }^{62}$ Cfr. Weil, Vol I, 4-10 / 4-15. Para examinar la evolución de esta institución pueden verse, principalmente, los siguientes casos: Moran v. Hong Kong \& Shanghai Banking Corp. (In re Deltacorp, Inc.), 179 B.R. 773, 777 (Bankr. S.D.N.Y. 1995) (sobre su character de "equitable doctrine"). Sobre sus presupuestos y límites en el campo del derecho concursal: Union Sav. Bank v. Augie/Restivo Baking Co., Ltd. (In re Augie/Restivo Baking Co., Ltd.), 860 F. 2d 515, 517 (2d Cir. 1988); Chem. Bank N.Y. Trust Co. v. Kheel, 369 F. 2d 845, 847 (2d Cir. 1966). Sobre los efectos patrimoniales sobre las entidades o sujetos involucrados pueden verse: James Talcott, Inc. v. Wharton (In re Continental Vending Mach. Corp.), 517 F. 2d 997, 1000 (2d Cir. 1975), cert. Denied, 424 U.S. 913 (1976); Stone v. Eacho (In re Tip Top Tailors, Inc.), 127 F. 2d 284, 289 (4 ${ }^{\text {th }}$ Cir.), cert denied, 317 U.S. 635 (1942); Mather v. G.K. Pipe Corp. (In re Moran Pipe \& Supply Co.), 130 B.R. 588, 593 (Bankr. E.D. Okla. 1991). Sobre su aplicabilidad a entidades afiliadas al deudor que no son deudoras o que sí lo son puede verse Feldman v. Trustees of Beck Indus. (In re Beck Indus.), 479 F. 2 d 410, 419 (2d Cir.), cert. denied, 414 U.S. 858 (1973).
} 


\section{Quaestio Iuris}

evolución jurídica se encuentra la aplicación de la doctrina del "piercing the corporate veil" en casos de grupos de sociedades $^{63}$. Asimismo está relacionada con la jurisprudencia que establece los requisitos o criterios para no considerar a las entidades como separadas ${ }^{64}$. Algunos autores y entidades han estudiado la vinculación entre la consolidación sustancial y estas técnicas de financiación ${ }^{65}$.

Basados en la problemática descripta en los casos citados precedentemente los tribunales han aplicado la consolidación cuando existe identificación entre las entidades involucradas en estas operaciones financieras, con la finalidad de evitar una amenaza o proporcionar algún beneficio para los acreedores ${ }^{66}$, también considerando si los acreedores trataron con las entidades en cuanto que estaban separadas o en cuanto estaban unidas en un grupo ${ }^{67}$. Todo esto dentro de un marco de excepcionalidad de la institución de la consolidación, que sólo puede utilizarse con moderación, por circunstancias perentorias o extraordinarias y como última solución posible frente a una confusión de patrimonios, siempre en beneficio de todos los acreedores ${ }^{68}$. Sin embargo ello no ha impedido afirmar la existencia de una tendencia liberal al aplicar la consolidación a los casos en que los tribunales reconocen la integración económica de los sujetos involucrados ${ }^{69}$, lo que implica la aplicación indudable de principios específicos aplicables a los grupos de sociedades económicamente integrados o unificados en su actuación económica conocido como "Entreprise Law"70.

En las relaciones entre las distintas entidades que forman parte de un grupo en el que se dan las negociaciones sobre financiación que estamos estudiando la doctrina destaca la documentación en la que se regula contractualmente el régimen que gobierna la necesaria separación de las mismas (las así llamadas "Separatedness Covenants"), entre el originator del "Bankruptcy-remote Vehicle" (SPV por sus siglas en inglés) y las restantes entidades integrantes del grupo económico ${ }^{71}$.

\footnotetext{
${ }^{63}$ En los primeros casos los tribunales aplicaron esta doctrina para consolidar entidades pertenecientes a un grupo económico cuando habían sido utilizadas como un "mere instrumentality" o "alter ego". Cfr., por ejemplo, Maule Indus. V. Gerstel, 232 F. $2 \mathrm{~d}$ 294, 296 ( $5^{\text {th }}$ Cir. 1956) y paradigmáticamente el caso Sampsell v. Imperial Paper \& Color Corp., 313 U.S. 215,217 (1941).

${ }^{64}$ Cfr. Fish v. East, 114 F. 2d 177, 191 (10 ${ }^{\text {th }}$ Cir. 1940); el ya citado caso Stone v. Eacho (In re Tip Top Tailors, Inc.), 127 F. 2 d 284, 289 ( $4^{\text {th }}$ Cir.), cert denied, 317 U.S. 635 (1942); In re Vecco Constr. Indus., 4 B.R. 407, 410 (Bankr. E.D. Va. 1980); In re DRW Prop. Co., 82, 54 B.R. 489, 494 (Bankr. N.D. Tex. 1985); In re Donut Queen, Ltd., 41 B.R. 706, 709-10 (Bankr. E.D.N.Y. 1984).

${ }^{65} \mathrm{Cfr}$. Committee on Bankruptcy and Corporate Reorganization of the Association of the Bar of the City of New York, Structured Financing Techniques, 50 Bus. Law. 527 (1995). Para la doctrina puede verse Patrick C. Sargent, Bankruptcy Remote Finance Subsidiaries: The Substantive Consolidation Issue, 44 Bus. Law. 1223 (1989).

${ }^{66}$ Cfr. Eastgroup Props. v. S. Motel Assocs., 935 F. 2d 245, 249 ( $11^{\text {th }}$ Cir. 1991) y Drabkin v. Midland-Ross Corp. (In re Auto-Train Corp.), 810 F. 2d 270, 276 (D.C. Cir. 1987).

${ }^{67}$ Cfr. Union Sav. Bank v. Augie/Restivo Baking Co., Ltd. (In re Augie/Restivo Baking Co., Ltd.), 860 F. 2d at 518 (2d Cir. 1988).

${ }^{68}$ Cfr., por ejemplo, Chem. Bank N.Y. Trust Co. v. Kheel, 369 F. 2 d 847 (2d Cir. 1966) y más modernamente In re Owens Corning, 419 F. 3d 195, 199-200 (3d Cir. 2005).

${ }^{69}$ Cfr., más recientemente, el caso In re F.A. Potts \& Co., 23 B.R. 569, 571 (Bankr. E.D. Pa. 1982).

${ }^{70}$ Cfr. Weil, Vol I, en 4-14 citan los siguientes fallos: In re Commercial Envelope Mfg. Co., 3 Bankr. Ct. Dec. (CRR) 647, 649 (S.D.N.Y. 1977); In re Standard Brands Paint Co., 154 B.R. 563, 572 (Bankr. C.D. Cal. 1993); In re Richton Int'l Corp., 12 B.R. 555, 557 (Bankr. S.D.N.Y. 1981) e In re Vecco Constr. Indus., 4 B.R. 407, 409 (Bankr. E.D. Va. 1980).

${ }^{71} \mathrm{Cfr}$. Weil, Vol I, en 4-14 los autores detallan las áreas en las que suele pactarse la necesaria separación entre las distintas entidades involucradas, a saber: la separación de la contabilidad general y financiera, la provisión de fondos dinerarios, los activos de cada una, el gobierno empresarial independiente, los pagos de pasivos con los respectivos fondos, la observancia de las formalidades vol.09, nº. 01, Rio de Janeiro, 2016.pp. 545-585 
Creación de la Bankruptcy-Remote Entity (SPV)

Además de aislar los activos que se pueden recibir del "originator" y, como consecuencia, de los riesgos de una posible quiebra del mismo, la securitización de activos implica por otra parte también la minimización de los riesgos de una quiebra de la Bankruptcy-Remote Entity (SPV). Esto es así en base a diversas razones, entre las que cabe mencionar las siguientes, a saber ${ }^{72}$ :

1. No obstante que en el marco de las normas del Chapter Eleven el SPV puede continuar sus operaciones como todo "Debtor-in-Possession"73, las mismas lo serán bajo el escrutinio del tribunal y eventualmente del Comité de acreedores.

2. Ese comité también evaluará la conveniencia de la reunión y venta de la mayor cantidad de bienes posible para obtener una mejora económica de los acreedores concursales.

3. El tribunal puede nombrar un Trustee independiente que haga una evaluación económica de la operación, también en el caso de que el proceso se convierta en uno regido por el Chapter Seven ${ }^{74}$.

4. En cualquiera de estos casos esto significa que cuando sea designado el Trustee, el mismo operará los negocios del SPV y suplantará a los miembros del directorio ${ }^{75}$.

No está muy claro cuáles son los sujetos abarcados por la norma como susceptibles de inclusión como SPV. Al respecto 11 U.S.C. \$109(a),(d) afirma que sólo una "Person" puede ser un deudor concursable conforme el Chapter Seven o el Chapter Eleven y a pesar de que una "Corporation" califica como "Person" conforme el código concursal", un "Nonbusiness Trust" no puede ser considerada como "Corporation", conforme la jurisprudencia $^{77}$, la que por otra parte ha reconocido que está dividida sobre el punto ${ }^{78}$.

La jurisprudencia ha reconocido deberes fiduciarios de los directores del SPV respecto de los accionistas de la entidad y de los acreedores ${ }^{79}$, incluso de los directores en el caso de la vecindad con la situación de la

societarias, el mantenimiento de unas relaciones independientes con las afiliadas ("at arm's length"), los pagos de salarios de sus empleados con fondos propios, la no garantización o asunción de obligaciones de otros sujetos integrantes, la atribución justa y razonable de costos, gastos y expensas originados en el propio funcionamiento, la utilización de documentación obligacional propia para las respectivas operaciones, el no otorgamiento de garantías colaterales a otras entidades y el mantenimiento de los vínculos como entidad separada.

${ }^{72}$ Cfr. Weil, Vol I, 4-15 / 4-20.

${ }^{73}$ Cfr. 11 U.S.C. $\$ \$ 1107$ (a), 1108

${ }^{74}$ Cfr. 11 U.S.C. $\$ \$ 701,702,704,1112$.

${ }^{75}$ Cfr. Commodity Futures Trading Comm'n v. Weintraub, 471 U.S. 343, 352-53 (1985)

${ }^{76}$ Cfr. 11 U.S.C. $\$ 101(41)$. No obstante se contrapone a esta última la norma del 11 U.S.C. \$102(3). No profundizamos aquí esta cuestión.

${ }^{77}$ Cfr. Shawmut Bank Conn. V. First Fidelity Bank (In re Secured Equip. Trust of Eastern Air Lines, Inc.), 38 F. 3d 86 (2d Cir. 1994). En otros casos la jurisprudencia habla de una "Entity". No profundizaremos esta cuestión aquí.

${ }^{78}$ Cfr. Shawmut Bank Conn. V. First Fidelity Bank (In re Secured Equip. Trust of Eastern Air Lines, Inc.), 38 F. 3d 86, at 89 (2d Cir. 1994).

${ }^{79}$ Cfr., por ejemplo, Katz v. Oak Indus., 508 A. 2 d 873 (Del. Ch. 1986). 
insolvencia, en los que ellos no son unos meros representantes de los accionistas sino que tienen deberes fiduciarios respecto de la sociedad y sus acreedores ${ }^{80}$.

Existen Estados como Delaware, en cuyos Business Trusts los directores pueden pactar una expansión o contracción de las cláusulas reguladoras de sus deberes fiduciarios en instrumentos de gobierno ${ }^{81}$.

\section{Valoración crítica del Chapter Eleven y su relación con los procedimientos de reestructuración extrajudiciales}

Luego del análisis de la temática tratada cabe realizar el siguiente juicio crítico, a saber:

1. La comparación entre los procedimientos establecidos por el Chapter Eleven del United States Code y las normas referidas a los procedimientos colectivos extrajudiciales, ("Out of Court Restructurings", "Workouts", "Extensions", “Compositions", etc.) en el derecho concursal norteamericano arroja como resultado en primer lugar un catálogo de los temas comprendidos en la elección alternativa de ambos procesos, referidos a sus ventajas e inconvenientes, concretados en la negociación con los principales prestamistas con preferencias, la negociación con los pequeños acreedores y la utilización de técnicas de financiación postconcursal. Este catálogo es importante porque fija la agenda de los operadores jurídicos en orden a arribar a las soluciones que el problema reclama, más allá de los buenos deseos e intenciones de las partes para solucionar la crisis económica.

2. En este trabajo he seguido la sistemática de los autores norteamericanos respecto de los tópicos tratados y en el modo de hacerlo, lo que tiene importantes diferencias sistemáticas en otros países del sistema continental europeo. La practicidad, eficiencia, eficacia y versatilidad de las soluciones del derecho estadounidense prima por sobre la supuesta perfección de los modelos lógico-jurídicos a aplicar a los casos de insolvencia.

3. La problemática regulada por las normas concursales en Estados Unidos hoy en día se ha expandido de modo amplio de manera tal que es necesaria una tarea de complementación profesional en materias tan dispares como, por ejemplo, los procesos extrajudiciales, los "Prepackaged Proceedings", los "Securities", el sector bancario, los procesos judiciales activos y pasivos del deudor, los derechos reales, los impuestos, las cuestiones medioambientales, laborales y jubilatorias y, finalmente, no sólo las cuestiones nacionales o domésticas sino también las multinacionales. Esto es novedoso por la multiplicidad de los supuestos prácticos a resolver, más allá de las normas puramente concursales. Esta forma de encarar los problemas proporciona perspectiva sistemática a la hora de encarar estos problemas.

\footnotetext{
${ }^{80}$ Cfr., por ejemplo, Credit Lyionnais Bank Nederland, N.V. v. Pathe Commc’ns Corp., No. 12150, 1991 WL 277613, at*36 n. 55 (Del. Ch. Dec. 30, 1991).

${ }^{81}$ Cfr. Weil, Vol I, cit., 4-22, nota 71.
} 
4. Dentro de los procedimientos no liquidativos los operadores jurídicos consideran como preeminente solución satisfactoria los arreglos extrajudiciales. Ellos constituyen métodos naturales y más convenientes que los procesos judiciales, los que sólo por excepción o subsidiariamente pueden prevenir la liquidación definitiva de la empresa. Ello surge conforme la jurisprudencia analizada y la experiencia alcanzada por los Estudios Jurídicos de abogados especializados en la materia concursal. Debe aquí destacarse que en los Estados Unidos las partes interesadas adjudican una marcada preponderancia a los sistemas extrajudiciales previos y preventivos de los judiciales a fin de solucionar un conflicto, lo que también se verifica en la materia concursal. Esta característica instrumental es digna de elogio y amerita un estudio en mayor profundidad en el futuro.

5. Estadísticamente se ha demostrado que aproximadamente la mitad de todas las reestructuraciones empresariales en los Estados Unidos son producto de un arreglo o composición extrajudicial, lo que es destacable y acredita el éxito de las vías extrajudiciales en ese país.

6. Como surge de la jurisprudencia americana la amplitud de la situaciones de crisis incluye casos de empresas saludables que tienen una abultada deuda financiera de corto y mediano plazo, hasta negocios que deben ser drásticamente reestructurados, reorganizados o diversificados o bien empresas que súbitamente se han visto envueltas en deudas originadas por responsabilidades patrimoniales derivadas de demandas colectivas tales como por ejemplo las originadas por sustancias cancerígenas, dispositivos intrauterinos, tabaco o drogas. Este doble enfoque es novedoso en orden a realizar un adecuado diagnóstico de la situación económico-financiera de la empresa y a fin de proporcionar una acertada elección de las soluciones prácticas pertinentes.

7. Los objetivos del legislador y de la jurisprudencia que promueven la evitación del acogimiento de la empresa a las normas concursales del Chapter Eleven en beneficio de lograr un arreglo extrajudicial de la crisis, constituye una acertada política legislativa en sus rasgos generales por cuanto la solución extrajudicial es, por una parte, más sencilla y práctica y por otro lado más rápida y barata.

8. El sistema extrajudicial norteamericano posee elevados niveles de eficacia ya que las partes interesadas están en condiciones de juzgar cuál de los dos procedimientos les es más beneficioso y en la mayoría de los casos ellas son reacias a acudir a los tribunales. Al parecer la cultura de los procesos extrajudiciales es más acusada en los Estados Unidos que en otros países del sistema continental europeo.

9. Como condición para esa eficacia de la vía extrajudicial es necesario por una parte lograr el afán colaborativo de todas las partes unidas por el común fin de lograr una solución preventiva de la liquidación y por otra parte la intención de todas ellas de brindar y utilizar lealmente toda la información necesaria para lograr esa solución, lejos de toda actitud fraudulenta. Debe destacarse esa colaboración ejercitada no sólo por la jurisprudencia sino también por la doctrina y los Estudios Jurídicos en pro de la 
consecución eficaz del arreglo extrajudicial de la deuda e indirectamente de la reorganización extrajudicial del deudor, lo que es encomiable. Esa colaboración se ha puesto de manifiesto particularmente en los Prepackaged Chapter Eleven, que no están regulados y a los que sólo se refieren las normas concursales de modo indirecto e implícito, con resultados exitosos.

10. Por lo general se considera que el arreglo extrajudicial es más beneficioso para la empresa que las posibilidades que ofrece el Chapter Eleven judicial, más allá de las dificultades que deben ser superadas para arribar al mismo. La negociación extrajudicial es más expeditiva y más económica que el proceso del Chapter Eleven.

11. No obstante lo anterior, el objeto del Chapter Eleven se ha revelado versátil, por cuanto no sólo pretende solucionar el problema económico-jurídico del estado de cesación de pagos o crisis, sino también porque logra una liquidación (incluso la parcial) en condiciones más ventajosas que otros procedimientos liquidativos del sistema federal estadounidense, como el del Chapter Seven, por ejemplo. Esto es novedoso por cuanto excede la sola finalidad del salvataje empresarial de este ordenamiento concursal preventivo y por otra parte porque pone de relieve que la solución liquidatoria no es tan traumática, problemática o negativa como pudiera considerarse idealmente en otras culturas jurídicas como la europea o la latinoamericana. En éstas últimas a veces idealiza el salvataje como la única mejor solución posible, bien que muy conveniente y altamente deseable siempre. Esto es lo que algunos autores han llamado el "mito de la empresa inmortal".

12. Las razones económicas para apoyar las normas e instituciones del Chapter Eleven han prevalecido frente a los costos y gastos del proceso, evitando innecesarias liquidaciones en detrimento patrimonial de los interesados.

13. Las alternativas previas al Chapter Eleven que han sido calificadas por algunos autores como "el mejor de los mundos posibles", esto es el "Prepackaged Chapter Eleven" y el "Prenegociated Chapter Eleven", pueden ser posibilidades válidas para solucionar extrajudicialmente los problemas económicos o financieros de la empresa sin necesidad de recurrir al procedimiento del Chapter Eleven, aunque todavía se encuentran en un período de desarrollo en sus institutos. Esta complementariedad entre la negociación preconcursal y el proceso concursal propiamente dicho ha sido eficaz para lograr la solución de la crisis o insolvencia en muchos casos. Será necesario en el futuro un estudio de derecho comprado sobre estas negociaciones preconcursales que posibiliten su aplicabilidad a otros ordenamientos jurídicos distintos de los países del Common Law, conforme las técnicas del derecho comparado.

14. Parte del éxito de los Prepackaged Chapter Eleven radica por una parte en la exigencia de la jurisprudencia respecto de la pertinencia e idoneidad de la información revelada por el deudor para la consecución de los objetivos del mismo. Esta información está referida a la descripción de los activos 
disponibles y su valuación, la información financiera que permita a los acreedores evaluar la propuesta y decidir la conformidad, el análisis del caso de liquidación y una acabada descripción del plan. Por otra parte ese éxito también radica en el compromiso de las partes contendientes sobre esa pertinencia y la seriedad de la información, en lo que se refiere a la adecuación entre la misma y la realidad negocial de las partes. La veracidad y sinceridad de la información facilita el entendimiento ulterior de las partes, al alejar toda posibilidad de fraude, quizá el principal enemigo de las soluciones extrajudiciales.

15. Una de las notables ventajas del "Pre-Packaged Plan" es su menor duración en el tiempo comparado con el proceso del Chapter Eleven y los menores costos de la negociación. Asimismo es destacable la versatilidad de este proceso extrajudicial, ya que, tanto si tiene éxito como si fracasara en el peor de los casos, lo negociado servirá ulteriormente para el proceso concursal del Chapter Eleven.

16. Respecto de la publicidad de la situación económico-financiera del deudor, los procesos extrajudiciales son más ventajosos que el proceso del Chapter Eleven porque aumentan la confianza de la propia empresa en la solución y la confianza de los terceros relacionados con ella y por ende aumenta las probabilidades de recuperación económica de la misma.

17. El peligro o dificultad para el éxito del Prepackaged Chapter Eleven está configurado por la identificación de las partes, la adecuada comunicación de los negocios, la suspensión del crédito por los acreedores y la posibilidad de algún acreedor de obtener una apertura concursal involuntaria del deudor.

18. Las tres principales técnicas usadas por los emisores de títulos de deuda de menor cuantía (también llamados "Junk Bonds") para reestructurar los pasivos en sede extrajudicial ("Tender Offers", "Exchange Offers" y "Consent Solicitations") han resultado exitosas para proveer al deudor de oxígeno financiero en el marco de una economía capitalista y con eficiencia. Cabe realizar en el futuro un estudio más profundo sobre la eficacia de estos institutos como instrumentos para lograr el financiamiento postconcursal del deudor en situación de crisis, tema que al parecer está poco estudiado en la literatura de los sistemas jurídicos de derecho continental europeo.

19. Otras técnicas de financiación del deudor en situación de cesación de pagos o crisis como la "Structured Financing" ("financiación estructurada") o la "Asset Securitization" ("Securitización de activos") han resultado útiles para arribar a soluciones en el corto y mediano plazo y con un costo menor que las técnicas de financiación tradicionales en el ámbito estadounidense. El régimen de este tipo de financiación ha recibido el respaldo de los tribunales que los han aplicado, con un conocimiento cabal de su funcionamiento, lo que es destacable como meritorio.

20. La unificación progresiva de los conceptos jurídicos y contables de la operatoria englobada en este tipo de técnicas de financiamiento han facilitado su instrumentación y la eficacia final de sus objetivos, gracias a los estándares contables establecidos por el Financial Accounting Standards Board (FASB) en al año 
2000 para este tipo de operaciones. Esta política puede ser útil para, por una parte, asegurar los beneficios del financiamiento postconcursal y, por otra parte, para aumentar la confianza en este tipo de técnicas financieras aplicables en otras jurisdicciones. Será oportuno en el futuro un mayor estudio de derecho comparado sobre la aplicabilidad de estas instituciones a fin de lograr una adecuada financiación postconcursal del deudor en otros sistemas jurídicos.

21. El marco de excepcionalidad de la institución de la consolidación, que debe utilizarse con moderación, por circunstancias compelentes o extraordinarias y como última solución posible frente a una confusión de patrimonios, y siempre en beneficio de todos los acreedores, ha otorgado seguridad jurídica y previsibilidad a las soluciones a las que ha arribado la jurisprudencia concursal y preconcursal, lo que es encomiable. Debe destacarse la necesidad de este uso adecuado de esa institución para que no devenga en inseguridad jurídica.

22. No obstante lo anterior, puede constatarse la existencia de una tendencia liberal al aplicar la consolidación a los casos en que los tribunales reconocen la integración económica de los sujetos involucrados, lo que implica la aplicación indudable de principios específicos aplicables a los grupos de sociedades económicamente integrados o unificados en su actuación económica conocido como "Entreprise Law".

23. Las dificultades conceptuales que han encontrado algunos tribunales respecto del sujeto que puede emitir securitizaciones no han impedido la existencia y funcionamiento del Bankruptcy-Remote Entity (SPV) como parte de las técnicas de financiación postconcursal. Esta institución puede servir de ejemplo de financiación eficaz y con menores costos, entre otras posibles. Será oportuno en el futuro un estudio de derecho comprado sobre su aplicabilidad en otros sistemas jurídicos distintos del Common Law.

24. La mayor debilidad del "Workout" está dada por el hecho de que cualquier acreedor rebelde o recalcitrante puede echar por tierra la negociación si exige más de lo que el deudor puede cumplir o embarga bienes esenciales para el giro comercial de la empresa.

25. A pesar de que el Chapter Eleven prevé mecanismos para imponer una solución consensuada por la mayoría de los acreedores a los acreedores resistentes y una valuación razonable de los bienes del deudor, las "Compositions" ofrecen algunas ventajas respecto del mismo, entre los que cabe mencionar los menores costos para el deudor, la no exposición pública a terceros de la situación económica del deudor y el no sometimiento del deudor al escrutinio y control judicial.

26. Cabe destacar que los procesos extrajudiciales de reestructuración de deuda y el proceso del Chapter Eleven no se excluyen mutuamente y son complementarios.

27. El concepto inglés de la falta de "Consideration" en los acuerdos extrajudiciales de reestructuración de deuda (de origen contractual), que habilita o impide la "Discharge" de las nuevas obligaciones asumidas 
por el deudor, luego de la negociación, ha mutado en el derecho de los Estados Unidos en los últimos ciento treinta años. El nuevo concepto de "Consideration" contiene las mutuas concesiones otorgadas por las partes negociadoras, favoreciendo así el acuerdo entre las partes. Esta evolución no sólo se ha debido a la jurisprudencia sino también a los autores y la legislación sobre contratos vigente.

28. Cabe destacar los beneficios del acuerdo extrajudicial comparándolos con los del Chapter Eleven, que se concretan en: una mayor confianza de los terceros en los futuros negocios de la empresa, la no publicidad de las operaciones empresariales, la menor litigiosidad, la mayor libertad de los directores para operar cambios en la dirección de la empresa, la mayor confidencialidad, la mayor celeridad de las soluciones, la mayor flexibilidad y simplificación de los procedimientos necesarios para alcanzar la reestructuración, la menor complejidad, la mayor expeditividad de las negociaciones con terceros y la disminución de los costos de negociación.

29. En el Workout, la actividad del directorio se centra en la preparación de un plan viable de reestructuración y de un plan de negocios y documentación de soporte del mismo que deberán ser presentados a los acreedores. Comparativamente, en el Chapter Eleven a esas tareas de gobierno societario se añaden otras, cuyo rol se hace más ambiguo y en cierta manera aleja a los directores de sus funciones naturales de dirección y gobierno.

30. Como causas del fracaso de la negociación extrajudicial cabe mencionar la desconfianza en las perspectivas económicas de futuro de la empresa, la desconfianza en la actuación de los miembros actuales del directorio, la desconfianza en los términos del "business plan" de la empresa, la perspectiva de los acreedores de recibir un mayor valor si se inicia un proceso de Chapter Eleven o se liquida la empresa, la posibilidad de obtener exitosas acciones de recomposición patrimonial mediante acciones judiciales contra transferencias fraudulentas realizadas a favor de otros acreedores, la necesidad de integrar al proceso un elevado número de acreedores para lograr una reestructuración empresarial y la gran complejidad de las cuestiones comprometidas en la negociación extrajudicial.

31. Respecto de las obligaciones tributarias, existen ventajas fiscales en el caso del Chapter Eleven que no existen en el caso de los Workouts.

32. Sobre la emisión y negociación con "Securities" al parecer no existen condiciones más o menos gravosas según que se trate de un estadio extrajudicial o judicial.

33. A pesar de que ordinariamente la mejor solución para la empresa en dificultades suele ser naturalmente la negociación extrajudicial, en algunos casos característicos es más conveniente la elección del proceso regulado en el Chapter Eleven a fin de lograr la reestructuración de la empresa y su pasivo. Ejemplos de esos casos son una alta litigiosidad en contra del deudor, los beneficios del "Automatic Stay" concursal, el cuestionamiento de la continuidad de los miembros del directorio, facilidades para obtener financiación 
postconcursal, mayor acceso al crédito comercial, la posibilidad de modificar sustancialmente los contratos empresariales más relevantes de la empresa y la posibilidad de imponer las soluciones concursales a los acreedores disconformes.

34. Últimamente han surgido otras formas extrajudiciales de negociación previas al Chapter Eleven que han resultado exitosas como el denominado "Prenegociated Chapter Eleven", institución actualmente "in fieri”.

\section{CONCLUSIONES ESENCIALES DE LA INVESTIGACION.}

A modo de conclusiones esenciales de la investigación cabe señalar lo siguiente:

1. Las instituciones del Chapter Eleven y de los procesos colectivos extrajudiciales constituyen vías alternativas o sucesivas válidas a fin de arribar a soluciones del estado de insolvencia o crisis de la empresa.

2. Ambos tipos de instituciones resultan idóneos desde el punto de vista práctico para lograr la superación de la insolvencia empresarial en los Estados Unidos. Esta experiencia es aplicable a países de otros sistemas jurídicos como el continental europeo, mediante las técnicas del derecho comprado.

3. Los escollos que a veces ha encontrado la jurisprudencia para lograr las soluciones de la insolvencia no han sido de tal entidad que impidan aplicar las soluciones correspondientes a los procesos extrajudiciales o judiciales.

4. La sencillez y simplicidad de los procesos extrajudiciales han permitido arribar a soluciones que en muchos casos han resultado ser más convenientes y eficaces que los procesos judiciales.

5. En la jurisprudencia no se han planteado graves cuestionamientos de fraude en los procesos extrajudiciales, quizá por un uso correcto de los mismos por las partes intervinientes, lejos de toda intencionalidad de fraude o con sus oportunas correcciones por las partes negociadoras, que siempre conservan la libertad de usar los remedios judiciales pertinentes.

\section{THE PROCESS OF U.S. BANKRUPTCY REORGANIZATION. (CHAPTER ELEVEN OF THE U. S. CODE). COMPARISON WITH OUT-OF-COURT RESTRUCTURINGS}

\footnotetext{
Abstract

This paper examines a part of the Bankruptcy law of the United States which deals with preventive solutions from liquidation in that country, which aims to preserve and virtually save the company in its economic and legal entity and is known by the name of its respective chapter, the Chapter Eleven of the Title Eleven of the United States Bankruptcy Code, a federal bankruptcy rule that includes many institutions in its various chapters.
} 
The Chapter Eleven establishes an insolvency process of "Reorganization" of the company in crisis ("Reorganization Procedure") in order to solve the economic or financial distress and keep the business alive and so prevent it from its compulsory liquidation, formal ultimate solution regulated in Chapter Seven of the federal Bankruptcy Code.

This paper is a comparison between of Chapter Eleven and Out-of-Court processes of business reorganization and restructuring of debt in the United States.

This work addresses the alternative choice of one of both procedures, their advantages and disadvantages, the different local (nonfederal) procedures of debt restructuring, the management of the company in crisis, the negotiations with major lenders, the negotiation with small creditors and the use of special financial techniques of the debtor.

In the final part of the paper the author makes a critical evaluation of the analized institutions.

Keywords: Bankruptcy Law - United States - Reorganization procedure - Chapter Eleven - Out of court restructurings.

\section{Referencias}

AAVV, Alan B. MORRISON (ed.), Fundamentals of American Law, Oxford University Press, Oxford, 1997.

AAVV. Accounting for Transfers and Servicing of Financial Assets and Extinguishments of Liabilities, Statement of Financial Accounting Standards n 140 (Financial Accounting Standards Bd. 2000) (FAS 140).

AAVV. David S. CLARK / Turgrul ANSAY (eds.), Introduction to the Law of the United States, Kluwer Law International, The Hague / London / New York, 2002.

AAVV. HARVARD LAW REVIEW ASSOCIATION (ED.). The Bluebook. A uniform system of Citation. 19० Ed. Cambrigde. Massachusetts, 2003.

AAVV. WEIL, GOTSHAL \& MANGES, LLP, Reorganizing Failing Businesses, A Comprehensive Review and Analysis of Financial Restructuring and Business Reorganization, Rev. Edition, American Bar Association, Chicago, 2006 (Dos volúmenes).

ADLER, Barry Ed., "Bankruptcy and RiskAllocation", 77 Cornell L. Rev. 439 (1992). "Financial and Political Theories of American Corporate Bankruptcy", 45 Stan. L. Rev. 311 (1993). "A World Without Debt", 72 Wash. U. L. Q. 811 (1994). "Finance's Theoretical Divide and the Proper Role of Insolvency Rules", 68 S. Cal. L. Rev. 401 (1994).

AGHION, Philippe / Oliver HART \& John MOORE, “The Economics of Bankruptcy Reform”, 8 J. L. Econ. \& Org. 523 (1992).

AGHION, Philippe et al., “The Economics of Bankruptcy Reform”, J.L. Econ \& Organization 523 (1992).

ALTMAN, Edward A., "Evaluating the Chapter 11 Bankruptcy Reorganization Process", 1 Colum. Bus. L. Rev. (1993).

AMERICAN BANKRUPTCY INSTITUTE, Comission to Study the Reform of Chapter 11: 2012-2014 Final Report and Recommendations. https://abiworld.app.box.eom/s/wircv5xv83aavl4dp4h . 
AYER, John D., "Bankruptcy as an Essentially Contested Concept: The Case of the One-Asset Case", 44 S.C.L. Rev. 863 (1993).

"Chapter 11: Uses and Consequences", 4 Am. Bankr. Inst. L. Rev. 493 (1996). $883(1994)$.

"Through Chapter 11 With Gun or Camera, But Probably Not Both: A Field Guide", 72 Wash U.L.Q.

BAIRD, Douglas G. / Thomas H. JACKSON / Barry E. ADLER, Bankruptcy, Cases, Problems and Materials, University Casebook Series, Rev. Foundation Press, New York, 3rd. Ed., 2001.

BAIRD, Douglas G., "Revisiting Auctions in Chapter 11", 36 J. L. \& Econ. 633 (1993).

The Elements of Bankruptcy, Foundation Press, New York, 4th Ed., 2006.

BANKRUPTCY CODE, Rules \& Official Forms, 2005 Law School Edition, Thomson West.

BARLIANT, Ronald / Dimitri G. Karcazes \& Anne M. Sherry, From Free-Fall to Free-For-All: The Rise of the Pre-Packaged Asbestos Bankruptcies, 12 Am. Bankr. Inst. L. Rev. 441 (2004).

BEBCHUK, Lucian Ayre, “A New Approach to Corporate Reorganizations”, 101 Harv. L. Rev. 775 (1986).

BHANDARI, Jagdeep S., Corporate Bankruptcy: Economic and Legal Perspectives, New York, 1996.

BLUM, Brian A., Bankruptcy and Debtor / Creditor, Examples and Explanations, Aspen Publishers, New York, 2006.

BLUMBERG, Philip, Problems in the bankruptcy or reorganization of parent and subsidiary corporations, including the law of corporate guaranties, Little, Brown \& Co., Boston, 1985 y supplement 2000.

BOWERS, James W., "Groping and Coping in the Shadow of Murphy's Law: Bankruptcy Theory and the Elementary Economics of Failure”, 88 Mich. L. Rev. 2097 (1990).

"Rehabilitation, Redistribution, of Dissipation: The Evidence for Choosing Among Bankruptcy Hypotheses", 72 Wash. U.L.Q.955 (1994).

"The Fantastic Wisconsylvania Zero-Bureaucratic Cost School of Bankruptcy Theory: A Comment", 91 Mich. L. Rev. 1773 (1993).

BRADLEY, Michael \& Michael ROSENZWEIG, “The Untenable Case for Chapter 11", 101 Yale L.J. 1043 (1992).

BRAUCHER, Jean, "Bankruptcy Reorganization and Economic Development", 23 Cap. U.L. Rev. 499 (1994).

BRUBAKER, Ralph, "One Hundred Years of Federal Bankruptcy Law and Still Clinging To An In Rem Model of Bankruptcy Jurisdiction”, 15 Bankr. Dev.J. 261 (1999).

CARLSON, David Gray, "Bankruptcy Theory and the Creditors' Bargain”, 61 U. Cin. L. Rev. 453 (1992).

COMMITTEE ON BANKRUPTCY AND CORPORATE REORGANIZATION OF THE ASSOCIATION OF THE BAR OF THE CITY OF NEW YORK, New Developments in Structured Finance, 56 Bus. Law. 95 (2000).

Structured Financing Techniques, 50 Bus. Law. 527 (1995). 
CRAMES, Michael J. / Herbert S. Edelman \& Andrew A. Kress, The Fundamentals of Bankruptcy and Corporate Reorganization, Juris Publishing, 2003.

EASTERBROOK, Frank H., "Is Corporate Bankruptcy Efficient?”, 27 J. Fin. Econ. 411 (1990).

EISENBERG, Theodore \& Lynn LOPUCKI, "Shopping For Judges: An Empirical Analysis of Venue Choice in Chapter 11 Reorganizations”, 84 Cornell L. Rev. 967 (1999).

EISENBERG, Theodore \& Shoichi TAGASHIRA, "Should We Abolish Chapter 11? The Evidence From Japan", 23 J. Legal Stud. 111 (1994).

EISENBERG, Theodore \& Stefan SUNDGREN, "Is Chapter 11 Too Favorable to Debtors? Evidence From Abroad", 82 Cornell L. Rev. 1532 (1997).

EISENBERG, Theodore, "Bankruptcy Law in Perspective”, 28 UCLA L. Rev. 953 (1981).

"The Unsecured Creditor in Reorganizations and the Nature of Security", 38 Vand. L. Rev. 931 (1985).

FARNSWORTH, E. Allan, Contracts, (2d ed. 1990).

FENNING, Lisa H., “The Future of Chapter 11: One View from the Bench”, 1993-1994 Ann. Surv. Bankr. L. 113.

FERRIELL, Jeff y Edward J. Janger, Understanding Bankruptcy, Matthew Bender \& Co., Inc. (Lexis Nexis Group), 2007.

FREY, Martin A., Introduction to Bankruptcy Law, Thomson / Delmar Learning, 2007.

FROST, Christopher W, "Bankruptcy Redistributive Policies and the Limits of the Judicial Process", 74 N. C. L. Rev. 75 (1995).

GILSON, Stuart C. et al., Troubled Debt Restructurings: An Empirical Study of Private Reorganization of Firms in Default, 27 J. Fin. Econ. 315, 345 (1990).

HERBERT, Michael J., Understanding Bankruptcy, Lexis Nexis, Matthew Bender, New York, 1995.

HOWARD, Margaret, Bankruptcy, Cases and Materials, American Casebook Series, Thomson / West Publishing, St. Paul, Minnesota, 2004.

JACKSON, Thomas H., "Bankruptcy, Nonbankruptcy Entitlements and the Creditors' Bargain", 91 Yale L. J. 857 (1982).

The logic and limits of bankruptcy law, Harvard University Press, Boston, 1986.

JOHNSTON, J. Bradley, “The Bankruptcy Bargain”, 65 Am. Bankr. L.J. 213 (1991).

JONES, Edith A., "Chapter 11: A Death Penalty for Debtor and Creditor Interests", 77 Cornell L. Rev. 1088 (1992).

KEATING, Daniel, “Offensive Uses of the Bankruptcy Stay”, 45 Vand. L. Rev. 71 (1991).

KLEE, Kenneth N., "Adjusting Chapter 11: Fine Tuning the Plan Process", 69 Am. Bankr. L. J. 551 (1995). 
KOROBKIN, Donald R, "Contractarianism and the Normative Foundations of Bankruptcy Law", 71 Tex. L. Rev. 541 (1993).

"Rehabilitating Values: A Jurisprudence of Bankruptcy", 91 Colum. L. Rev. 717 (1991).

"The Unwarranted Case Against Corporate Reorganization: A Reply to Professors Bradley and Rosenzweig", 78 Iowa L. Rev. 669 (1993).

KRUGER, Lewis, Understanding the Basics of Business Bankruptcy and Reorganization 2008, Practicing Law Institute, New York, 2000 et sig.

LOPUCKI, Lynn M., "Contract Bankruptcy: A Reply to Alan Schwartz", 109 Yale L. J. 317 (1999). "Bankruptcy Contracting Revised: A Reply to Alan Schwartz's New Model", 109 Yale L.J. 365 (1999). "Chapter 11: An Agenda for Basic Reform", 69 Am. Bankr. L.J. 573 (1995).

"The Trouble With Chapter 11", 1993 Wis. L. Rev. 729. (1992). "Strange Visions in a Strange World: A Reply to Professors Bradley and Rosenzweig", 91 Mich. L. Rev. 79

MACDONALD, Mark E. et al., "Chapter 11 as a Dynamic Evolutionary Learning Process in a Market with Fuzzy Values”, 1993-1994 Ann. Surv. Bankr. L. 1.

MIGUENS, Héctor José, "El proceso de Reorganización Concursal de Estados Unidos. (Chapter Eleven del U. S. Code). Introducción y aspectos generales", en Anuario de Derecho Concursal (España), vol 34 (enero 2015) ps. $477-543$.

PONOROFF, Lawrence, "Enlarging the Bargaining Table: Some Implications of the Corporate Stakeholder Model for Federal Bankruptcy Proceedings”, 23 Cap. U.L. Rev. 441 (1994).

RASMUSSEN, Robert K. \& David A. SKELL, Jr., "The Economic Analysis of Corporate Bankruptcy Law”, 3 Am. Bankr. Inst. L. Rev. 85 (1995).

RASMUSSEN, Robert K. "Debtor's Choice: A Menu Approach to Corporate Bankruptcy”, 71 Tex. L. Rev. 51 (1992).

"The Efficiency of Chapter 11", 8 Bankr. Dev.J. 319 (1991).

"The Ex Ante Effects of Bankruptcy Reform on Investment Incentives", 72 Wash U.L.Q. 1159 (1994).

RESNICK, Alan N., Bankruptcy Law Manual, Thomson / West, 5th. Ed., 2002- .

ROE, Mark J., "Bankruptcy and Debt: A New Model for Corporate Reorganizations", 83 Colum. L. Rev. 527 (1983). 2007.

Bankruptcy and Corporate Reorganization, Legal and Financial Materials, Foundation Press, New York,

RUSCH, Linda J., "Bankruptcy Reorganization Jurisprudence: Matters of Belief, Faith and Hope - Stepping into the Fourth Dimension”, 55 Mont. L. Rev.9 (1994).

SARGENT, Patrick C., Bankruptcy Remote Finance Subsidiaries: The Substantive Consolidation Issue, 44 Bus. Law. 1223 (1989). 
SCARBERRY, Mark S. / Kenneth N. KLEE / Grant W. NEWTON / Steve H. NICKES, Business Reorganization in Bankruptcy, Cases and Materials, Thomson / West Publishing, 3rd. Ed., 2006.

SCHWARCZ, Steven L., "Rethinking Freedom of Contract: A Bankruptcy Paradigm", 77 Tex. L. Rev. 515 (1999).

SCHWARTZ, Alan, "Bankruptcy Workouts and Debt Contracts", 36 J. L. \& Econ. 595 (1993).

"Contracting About Bankruptcy", 13 J. L. \& Econ. 595 (1993).

SKEEL, Jr., David A. "Rethinking the Line Between Corporate Law and Corporate Bankruptcy", 72 Tex. L. Rev. 471 (1994).

SKEEL, Jr., David A., "Markets, Courts and the Brave New World of Bankruptcy Theory”, 1993 Wis. L. Rev. 465.

SMYSER, Kathryn, "Going Private and Going Under: Leveraged Buyouts and the Fraudulent Conveyance Problem”, 63 Ind. L.J. 781 (1988).

SULLIVAN, Teresa / Elisabeth WARREN / Jay L. WESTBROOK, As We Forgive our Debtors: Bankruptcy and Consumer Credit in America, Oxford University Press, Oxford, 1989 (hay edición 1991).

TABB, Charles J. / Ralph BRUBAKER, Bankruptcy Law: Principles, Policies and Practice, Lexis Nexis, 2nd Ed., 2006.

Bankruptcy Anthology, Anderson Publishing Co., Cincinnati, Ohio, 2002.

“The Future of Chapter 11", 44 S. C. L. Rev. 791, 804 (1993).

The Law of Bankruptcy, The Foundation Press, Westbury, New York, 1997.

The Law of Bankruptcy, The Foundation Press, Inc., Westbury, New York, 1997, ps. 25-27.

WARREN, Elisabeth / Jay Lawrence WESTBROOK, The Law of Debtors and Creditors, Text, Cases and Problems, Wolters Kluwer Law and Business, Austin / Boston / Chicago / New York / The Netherlands, 6th Ed., 2009.

The Law of Debtors and Creditors, Text, Cases and Problems, 6th Ed., Wolters Kluwer, Austin, Boston, Chicago, New York, The Netherlands, 2009.

WARREN, Elisabeth, "Bankruptcy Policy”, 54 U. Chi. L. Rev. 775 (1987).

"Bankruptcy Policymaking in an Imperfect World", 92 Mich. L. Rev. 336 (1993).

"The Untenable Case for the Repeal of Chapter 11", 102 Yale L.J. 437 (1992).

Chapter 11: Reorganizing American Businesses, Aspen Publishers, Wolters Kluwer Law \& Business, Austin / Boston / Chicago / New York / The Netherlands, 2008.

WARREN, William D. / Daniel J. BUSSEL, Bankruptcy, University Casebook Series, 4th ed., Foundation Press, New York, 2002.

WEISS, Lawrence A., "Bankruptcy Resolution: Direct Costs and Violation of Priority of Claims", 27 J. Fin. Econ. $285(1990)$.

WHITE, Michelle J., "Does Chapter 11 Save Economically Inefficient Firms?” 72 Wash. U.L.Q. 1319 (1994).

"Corporate Bankruptcy as a Filtering Device: Chapter 11 Reorganiations and Out-of-Court Deb Restructurings”, 10 J. L. Econ. \& Org. 268 (1994).

WHITFORD, William C.,'What's Right About Chapter 11", 72 Wash. U.L.Q. 1379 (1994). 
WILLIAMS, Winton E., Resolving the Creditor's Dilemma: An Elementary Game. Theoretic Analysis of the Causes and Cures of Counterproductive Practices in the Collection of Consumer Debt, 48 Fla. L. Rev. 607 (1996).

\section{Jurisprudencia}

BLACKFORD V. COMMENCIAL CREDIT CORP., 263 F. 2d 97, 106 (5th Cir.), cert denied, 361 U.S. 825 (1959).

CAPLAN V. UNIMAX HOLDINGS, Corp., 591 N.Y.S. 2 d 28 (N.Y. App. Div. 1992).

CASTLE ROCK INDUS. BANK V. S.O.A.W. ENTERS. (IN RE S.O.A.W. ENTERS), 32 B.R. 279 (Bankr. W.D. Tex. 1983).

CHEM. BANK N.Y. TRUST CO. V. KHEEL, 369 F. 2d 845, 847 (2d Cir. 1966).

CHEM. BANK N.Y. TRUST CO. V. KHEEL, 369 F. 2 d 847 (2d Cir. 1966).

COMMODITY FUTURES TRADING COMM’N V. WEINTRAUB, 471 U.S. 343, 352-53 (1985).

CREDIT LYIONNAIS BANK NEDERLAND, N.V. V. PATHE COMMC'NS CORP., No. 12150, 1991 WL 277613, at*36 n. 55 (Del. Ch. Dec. 30, 1991).

DRABKIN V. MIDLAND-ROSS CORP. (IN RE AUTO-TRAIN CORP.), 810 F. 2d 270, 276 (D.C. Cir. 1987).

EASTGROUP PROPS. V.S. MOTEL ASSOCS., 935 F. 2d 245, 249 ( $11^{\text {th }}$ Cir. 1991).

FELDMAN V. TRUSTEES OF BECK INDUS. (IN RE BECK INDUS.), 479 F. 2d 410, 419 (2d Cir.), cert. denied, 414 U.S. 858 (1973).

FIREMAN'S FUND INS, COOS. V. GROVER (IN RE THE WOODSON CO.), 813 F. $2 \mathrm{~d} 266,272$ ( $9^{\text {th }}$ Cir. 1987).

FISH V. EAST, 114 F. 2d 177, 191 (10 ${ }^{\text {th }}$ Cir. 1940).

FOAKES V. BEER, L.R. 9 A.C. 605 (H.L. 1884), de la House of Lords.

FOX V. PECK IRON \& METAL CO, 25 B.R. 674, 690 (BANKR. S.D.CAL. 1982) Y CENT. DISCOUNT CO. V.DEP’T OF REVENUE, 94 N.W.2d 805 (Mich. 1959).

GOLDEN PLAN, 829 F. 2D 705 Y RECHNITZER V. BOYD (IN RE EXECUTIVE GROWTH INVS., INC.) 40 B.R. 417, 422 (Bankr. C.D. Cal. 1984).

HARTFORD FIRE INSURANCE CO. V. FEDERATED DEPARTMENT STORES, Inc. 723 F. Supp. 976 (S.D.N.Y. 1989).

IN RE COMMERCIAL ENVELOPE MFG. CO., 3 Bankr. Ct. Dec. (CRR) 647, 649 (S.D.N.Y. 1977). 
IN RE DONUT QUEEN, LTD., 41 B.R. 706, 709-10 (Bankr.E.D.N.Y. 1984).

IN RE DRW PROP. CO., 82, 54 B.R. 489, 494 (Bankr. N.D. Tex. 1985).

IN RE F.A. POTTS \& CO., 23 B.R. 569, 571 (Bankr. E.D. Pa. 1982).

IN RE FF HOLDINGS CORPORATION, Case n. 98 -37 (Bankr. Del. January 7, 1998) (Farnin C.J.).

IN RE IWO HOLDINGS, Inc., nº 05-100009 (PJW) (Bankr. D. Del. Feb. 9, 2005).

IN RE MAGELLAN HEALTH SERV., Inc., nº 03-40515 (PCB), (Bankr. S.D.N.Y. Oct 8, 2003).

IN RE OWENS CORNING, 419 F. 3d 195, 199-200 (3d Cir. 2005).

IN RE RICHTON INT’L CORP., 12 B.R. 555, 557 (Bankr. S.D.N.Y. 1981).

IN RE SOUTHLAND CORP., 124 B.R. 211, 213 (Bankr. N. D. Tex. 1991).

IN RE STANDARD BRANDS PAINT CO., 154 B.R. 563, 572 (Bankr. C.D. Cal. 1993).

IN RE VECCO CONSTR. INDUS., 4 B.R. 407, 409 (Bankr. E.D. Va. 1980).

IN RE VECCO CONSTR. INDUS., 4 B.R. 407, 410 (Bankr. E.D. Va. 1980).

JAMES TALCOTT, INC. V. WHARTON (IN RE CONTINENTAL VENDING MACH. CORP.), 517 F. $2 \mathrm{~d}$ 997, 1000 (2d Cir. 1975), cert. Denied, 424 U.S. 913 (1976).

KATZ V. OAK INDUS., 508 A. 2 d 873 (Del. Ch. 1986).

KATZ V. OAK INDUSTRIES, 508 A. 2d 873, 880 (Del. Ch. 1986).

MAJOR'S FURNITURE MART, INC. V. CASTLE CREDIT CORP., 602 F. 2d 538, 542-45 (3d Cir. 1979).

MATHER V. G.K. PIPE CORP. (IN RE MORAN PIPE \& SUPPLY CO.), 130 B.R. 588, 593 (Bankr. E.D. Okla. 1991).

MAULE INDUS. V. GERSTEL, 232 F. 2d 294, 296 ( $5^{\text {th }}$ Cir. 1956).

METROPOLITAN LIFE INSURANCE CO. V. RJR NABISCO, Inc., 906 F. 2 d 884 (2d Cir. 1990).

MORAN V. HONG KONG \& SHANGHAI BANKING CORP. (IN RE DELTACORP, INC.), 179 B.R. 773, 777 (Bankr. S.D.N.Y. 1995).

RECHNITZER V. BOYD (IN RE EXECUTIVE GROWTH INVS., INC.), 40 B.R. 417, 422 (Bankr. C.D. Cal. 1984).

SAMPSELL V. IMPERIAL PAPER \& COLOR CORP., 313 U.S. 215, 217 (1941). 
SHAWMUT BANK CONN. V. FIRST FIDELITY BANK (IN RE SECURED EQUIP. TRUST OF EASTERN AIR LINES, INC.), 38 F. 3d 86 (2d Cir. 1994).

SHAWMUT BANK CONN. V. FIRST FIDELITY BANK (IN RE SECURED EQUIP. TRUST OF EASTERN AIR LINES, INC.), 38 F. 3d 86, at 89 (2d Cir. 1994).

SIMONS V. COGAN, 542 A. 2d 785, 787 (Del. Ch. 1987).

STONE V. EACHO (IN RE TIP TOP TAILORS, INC.), 127 F. 2d 284, 289 ( $4^{\text {th }}$ Cir.), cert denied, 317 U.S. 635 (1942).

Straton v. New, 283 U.S. 318 (1931), de la Corte Suprema Federal.

UNION SAV. BANK V. AUGIE/RESTIVO BAKING CO., LTD. (IN RE AUGIE/RESTIVO BAKING CO., LTD.), 860 F. 2d 515, 517 (2d Cir. 1988).

UNION SAV. BANK V. AUGIE/RESTIVO BAKING CO., LTD. (IN RE AUGIE/RESTIVO BAKING CO, LTD.), 860 F. 2 d at 518 (2d Cir. 1988).

UNITED ARTISTS THEATRE CO. V. WALTON, 315 F. 3d 217, 224 n. 5 (3d Cir. 2003).

VAN GEMERT V. BOEING CO., 520 F. 2 d 1373 (2d Cir.), cert. denied, 423 U.S. 947 (1975).

WEST PICO FURNITURE CO. V. PAC. FIN. LOANS, 469 P. 2 d 665 (Cal. 1970).

Trabalho enviado em 03 de fevereiro de 2016.

Aceito em 03 de fevereiro de 2016. 